\title{
SPECTRAL ASYMPTOTICS FOR COMPACT SELF-ADJOINT HANKEL OPERATORS
}

\author{
ALEXANDER PUSHNITSKI AND DMITRI YAFAEV
}

To the memory of Yura Safarov

\begin{abstract}
We describe large classes of compact self-adjoint Hankel operators whose eigenvalues have power asymptotics and obtain explicit expressions for the coefficient in front of the leading term. The results are stated both in the discrete and continuous representations for Hankel operators. We also elucidate two key principles underpinning the proof of such asymptotic relations. We call them the localization principle and the symmetry principle. The localization principle says that disjoint components of the singular support of the symbol of a Hankel operator make independent contributions into the asymptotics of eigenvalues. The symmetry principle says that if the singular support of a symbol does not contain the points 1 and -1 in the discrete case (or the points 0 and $\infty$ in the continuous case), then the spectrum of the corresponding Hankel operator is asymptotically symmetric with respect to the reflection around zero.
\end{abstract}

\section{INTRODUCTION}

1.1. Localization and symmetry principles. Hankel operators admit various unitary equivalent descriptions. First we recall the definition of Hankel operators on the Hardy class $H^{2}(\mathbb{T})$. Let $\mathbb{T}$ be the unit circle in the complex plane, equipped with the normalized Lebesgue measure $d m(\mu)=(2 \pi i \mu)^{-1} d \mu, \mu \in \mathbb{T}$. The Hardy class $H^{2}(\mathbb{T}) \subset L^{2}(\mathbb{T})$ is defined in the standard way as the subspace spanned by the functions $1, \mu, \mu^{2}, \ldots$ in $L^{2}(\mathbb{T})$. Let $P_{+}: L^{2}(\mathbb{T}) \rightarrow H^{2}(\mathbb{T})$ be the orthogonal projection onto $H^{2}(\mathbb{T})$, and let $W$ be the involution in $L^{2}(\mathbb{T})$ defined by $(W f)(\mu)=$ $f(\bar{\mu})$. For a function $\omega \in L^{\infty}(\mathbb{T})$, the Hankel operator $H(\omega)$ with the symbol $\omega$ is defined on the Hardy class $H^{2}(\mathbb{T})$ by the relation

$$
H(\omega) f=P_{+}(\omega W f), \quad f \in H^{2}(\mathbb{T}) .
$$

Background information on the theory of Hankel operators can be found in the books [7, 8].

In this paper, we are interested in self-adjoint Hankel operators. Thus, we will always assume that the symbol $\omega$ satisfies the symmetry condition

$$
\overline{\omega(\mu)}=\omega(\bar{\mu}), \quad \mu \in \mathbb{T} .
$$

2010 Mathematics Subject Classification. 47B06, $47 \mathrm{~B} 35$.

Key words and phrases. Power asymptotics of eigenvalues, symbol, singular support, the localization principle, the symmetry principle, oscillating kernels. 
It is well known that $H(\omega)$ is bounded if $\omega \in L^{\infty}(\mathbb{T})$ and that $H(\omega)$ is compact if $\omega$ is continuous. Moreover, if $\omega \in C^{\infty}(\mathbb{T})$, then the eigenvalues of $H(\omega)$ go to zero faster than any power of $n^{-1}$ as $n \rightarrow \infty$. Conversely, the singularities of $\omega(\mu)$ are responsible for the power-like decay of eigenvalues or even for the appearance of the continuous spectrum.

The first result in this direction is due to S. R. Power [9] who considered the essential spectrum of $H(\omega)$ for piecewise continuous symbols $\omega$. The structure of the absolutely continuous spectrum was later described by J. S. Howland [4]. Although the assumptions of [9] and [4] are slightly different, in both cases $\omega$ has jump discontinuities on the unit circle; the essential (resp. absolutely continuous) spectrum can be described in terms of these jumps. It turns out that the contributions of different jumps of $\omega$ (i.e. the jumps located at different points of the unit circle) to the essential spectrum are independent of each other. We call this fact the localization principle. Further, observe that under the symmetry condition (1.2), the singularities of $\omega$ can be located (i) at the points +1 and -1 and (ii) at pairs $(\zeta, \bar{\zeta})$ of complex conjugate points on the unit circle. It turns out that the contributions of the jumps to the essential spectrum in cases (i) and (ii) are qualitatively different. More precisely, the jumps of $\omega(\mu)$ at the points $\mu= \pm 1$ yield the intervals $\left[0, \kappa_{ \pm}\right]$(with $\kappa_{ \pm}$determined by the size of the jumps) of the essential spectrum, while the jumps at each pair $(\zeta, \bar{\zeta})$ of complex conjugate points yield symmetric intervals $[-\kappa, \kappa]$. The last assertion is natural to call the symmetry principle.

Our goal is to find the asymptotic formulas for eigenvalues for wide classes of compact self-adjoint Hankel operators. To that end, we state both the localization principle and the symmetry principle in a rather general setting adapted to the study of the discrete spectrum. In this paper we are interested in Hankel operators with the power-like asymptotics of eigenvalues. For Hankel operators $H(\omega)$ realized in the space $H^{2}(\mathbb{T})$ by formula (1.1), such behavior occurs for symbols $\omega(\mu)$ with logarithmic singularities. This means that $\omega(\mu)$ is continuous at singular points $\zeta$ but the rate of convergence $\omega(\mu)-\omega(\zeta) \rightarrow 0$ as $\mu \rightarrow \zeta$ is logarithmic; thus, $\omega$ does not satisfy the Hölder condition with any positive exponent.

Before going into details, we describe another representation for Hankel operators as "infinite matrices".

1.2. Matrix Hankel operators. Let $\omega \in L^{\infty}(\mathbb{T})$, and let the operator $H(\omega)$ be defined by formula (1.1). The "matrix elements" of $H(\omega)$ in the orthonormal basis $\left\{\mu^{j}\right\}_{j=0}^{\infty}$ in $H^{2}(\mathbb{T})$ are

$$
\left(H(\omega) \mu^{j}, \mu^{k}\right)_{L^{2}(\mathbb{T})}=\widehat{\omega}(j+k), \quad j, k \geq 0,
$$

where $\widehat{\omega}$ are the Fourier coefficients of $\omega$,

$$
\widehat{\omega}(j)=\int_{\mathbb{T}} \omega(\mu) \mu^{-j} d m(\mu), \quad j \in \mathbb{Z} .
$$


This gives the standard "matrix representation" for Hankel operators in the space $\ell^{2}\left(\mathbb{Z}_{+}\right)$.

It will be convenient to introduce some notation related to this representation. For a sequence $\{h(j)\}_{j=0}^{\infty}$ of complex numbers, the Hankel operator $\Gamma(h)$ in the space $\ell^{2}\left(\mathbb{Z}_{+}\right)$is formally defined by the "infinite matrix" $\{h(j+k)\}_{j, k=0}^{\infty}$, that is,

$$
(\Gamma(h) u)(j)=\sum_{k=0}^{\infty} h(j+k) u(k), \quad u=\{u(j)\}_{j=0}^{\infty} \in \ell^{2}\left(\mathbb{Z}_{+}\right) .
$$

Of course, $\Gamma(h)$ is symmetric if the sequence $h$ is real-valued. By (1.3), a Hankel operator $\Gamma(h)$ is unitarily equivalent to $H(\omega)$ if and only if

$$
\widehat{\omega}(j)=h(j), \quad j \geq 0 ;
$$

in this case $\omega$ is called a symbol of $\Gamma(h)$. Since (1.5) involves only $j \geq 0$, a symbol is not uniquely defined. By Nehari's theorem [6], the operator $\Gamma(h)$ is bounded if and only if relation (1.5) is satisfied for some some $\omega \in L^{\infty}(\mathbb{T})$.

Suppose that, for some $\alpha \geq 0$ and $j \rightarrow \infty$,

$$
h(j)=\left(\mathrm{b}_{1}+\mathrm{b}_{-1}(-1)^{j}+2 \sum_{\ell=1}^{L} b_{\ell} \cos \left(\varphi_{\ell} j-\psi_{\ell}\right)\right) j^{-1}(\log j)^{-\alpha}+\text { error term }
$$

where $\varphi_{1}, \ldots, \varphi_{L} \in(0, \pi)$ are distinct numbers and $\psi_{1}, \ldots, \psi_{L}$ as well as $\mathrm{b}_{-1}, \mathrm{~b}_{1}, b_{1}, \ldots, b_{L}$ are arbitrary real numbers. It can be shown that the singular support of the symbol $\omega(\mu)$ of the corresponding Hankel operator $\Gamma(h)$ (without the error term in (1.6) ) consists of the points \pm 1 (if $b_{ \pm 1} \neq 0$ ) and the pairs $\left(e^{i \varphi_{\ell}}, e^{-i \varphi_{\ell}}\right), \ell=1, \ldots, L$. If $\alpha=0$, then $\omega(\mu)$ has jumps at these points. This implies (see [4] and [10]) that the absolutely continuous spectrum of $\Gamma(h)$ equals

$$
\operatorname{spec}_{\mathrm{ac}}(\Gamma(h))=\left[0, \pi \mathrm{b}_{1}\right] \cup\left[0, \pi \mathrm{b}_{-1}\right] \cup \bigcup_{\ell=1}^{L}\left[-\pi b_{-\ell}, \pi b_{\ell}\right]
$$

(each of the intervals in the right-hand side yields the absolutely continuous spectrum of multiplicity one).

In the case $\alpha>0$, the singularities of $\omega(\mu)$ are weaker so that the operators $\Gamma(h)$ are compact, and the decay of their eigenvalues is determined by these singularities. Let us describe a typical result of this paper. For a compact self-adjoint operator $\Gamma$, let us denote by $\left\{\lambda_{n}^{+}(\Gamma)\right\}_{n=1}^{\infty}$ the non-increasing sequence of positive eigenvalues of $\Gamma$ and set $\lambda_{n}^{-}(\Gamma)=\lambda_{n}^{+}(-\Gamma)$. We show that

$$
\lambda_{n}^{ \pm}(\Gamma(h))=a^{ \pm} n^{-\alpha}+o\left(n^{-\alpha}\right), \quad n \rightarrow \infty, \quad \alpha>0,
$$

where

$$
a^{ \pm}=\varkappa(\alpha)\left(\left(\mathrm{b}_{-1}\right)_{ \pm}^{1 / \alpha}+\left(\mathrm{b}_{1}\right)_{ \pm}^{1 / \alpha}+\sum_{\ell=1}^{L}\left|b_{\ell}\right|^{1 / \alpha}\right)^{\alpha}
$$


$b_{ \pm}=(|b| \pm b) / 2$, and the numerical coefficient $\varkappa(\alpha)$ can be expressed in terms of the Beta function,

$$
\varkappa(\alpha)=2^{-\alpha} \pi^{1-2 \alpha}\left(B\left(\frac{1}{2 \alpha}, \frac{1}{2}\right)\right)^{\alpha} .
$$

Thus both in the theory of the continuous spectrum (formula (1.7)) and in the theory of the discrete spectrum (formula (1.9)) the contributions of different terms in (1.6) are independent of each other. So it is natural to use the term the localization principle for this phenomenon.

Observe that the first two terms in the right-hand side of (1.6) contribute to the leading term of the asymptotics of $\lambda_{n}^{+}(\Gamma(h))$ (resp. of $\lambda_{n}^{-}(\Gamma(h))$ ) only if the coefficients $b_{1}$ or $b_{-1}$ are positive (resp. negative). On the other hand, in both continuous and discrete cases, the oscillating terms in (1.6) yield symmetric contributions to the continuous spectrum of $\Gamma(h)$ and to the leading term of the asymptotics of the eigenvalues $\lambda_{n}^{ \pm}(\Gamma(h))$. It is natural to call this phenomenon the symmetry principle.

1.3. Related work. This paper can be considered as a continuation of our previous work [12] where Hankel operators $\Gamma(h)$ with matrix elements (1.6) were studied for $\alpha>0$ under the assumption that $b_{1}=\cdots=b_{L}=0$; in this case $h(j)$ does not contain the oscillating terms. Extending this to the case of non-zero coefficients $b_{1}, \ldots, b_{L}$ turns out to be a non-trivial problem. In fact, we had to isolate and formalise both the localization and the symmetry principles for compact self-adjoint Hankel operators precisely in order to handle this situation.

We note also our paper [13] where Hankel operators $\Gamma(h)$ corresponding to the sequences

$$
h(j)=\left(\sum_{\ell=1}^{L} b_{\ell} \zeta_{\ell}^{-j}\right) j^{-1}(\log j)^{-\alpha}+\text { error term, } \quad j \rightarrow \infty, \quad \alpha>0,
$$

were considered. Here $\zeta_{1}, \ldots, \zeta_{L} \in \mathbb{T}$ are distinct points (not necessarily complex conjugate pairs of points) and $b_{1}, \ldots, b_{L}$ are any complex coefficients so that the operators $\Gamma(h)$ need not be self-adjoint. In [13], we studied singular values $s_{n}(\Gamma(h))$ of the operator $\Gamma(h)$ and obtained the asymptotic formula

$$
s_{n}(\Gamma(h))=a n^{-\alpha}+o\left(n^{-\alpha}\right), \quad n \rightarrow \infty,
$$

with the coefficient

$$
a=\varkappa(\alpha)\left(\sum_{\ell=1}^{L}\left|b_{\ell}\right|^{1 / \alpha}\right)^{\alpha} .
$$

The proof of this assertion in [13] used our result of [12] and also required the localization principle for singular values of Hankel operators. This principle allowed us to separate the contributions of different terms in the right-hand side of (1.11).

We finally mention the fundamental paper [5] where the spectra of all bounded self-adjoint Hankel operators were characterized in terms of a certain balance of their positive and negative parts. In particular, the spectra of compact Hankel operators were characterized by the two conditions: (i) the multiplicities of the 
eigenvalues $\lambda$ and $-\lambda$ do not differ by more than one; (ii) if the point $\lambda=0$ is an eigenvalue, then necessarily it has infinite multiplicity. The first of these conditions is similar in spirit to the asymptotic formulas (1.8), (1.9) for the eigenvalues, but of course neither of these two results implies the other one.

1.4. Main ideas of the approach. The results of [12, 13] are the basis for our proof of the relations (1.8), (1.9). The other two ingredients are the localization and the symmetry principles for eigenvalues.

The localization principle allows us to separate the contributions of different terms in the right-hand side of (1.6). In a more general setting it says that the contributions of disjoint components of the singular support of $\omega$ (denoted sing supp $\omega$ ) to the asymptotics of the eigenvalues of $H(\omega)$ are independent of each other. The precise statement is Theorem 3.2 below. This principle allows one to split the singular support of $\omega$ into disjoint pieces and to study the operators corresponding to each piece separately. In our previous work [13] we discussed localization principle for singular values of (not necessarily self-adjoint) Hankel operators. The localization principle for eigenvalues requires some new operator theoretic input, which is stated here as Theorem 2.2 .

The symmetry principle is needed to treat Hankel operators $\Gamma(h)$ with the oscillating matrix elements $h(j)=\cos (\varphi j-\psi) j^{-1}(\log j)^{-\alpha}$. It shows that asymptotically the sequence $s_{n}(\Gamma(h))$ of singular values is "shared equally" between the sequences $\lambda_{n}^{+}(\Gamma(h))$ and $\lambda_{n}^{-}(\Gamma(h))$ of positive and negative eigenvalues. So given the asymptotic formula for $s_{n}(\Gamma(h))$ obtained in [13], we get a formula for $\lambda_{n}^{ \pm}(\Gamma(h))$. More generally, the symmetry principle says that if sing $\operatorname{supp} \omega$ does not contain 1 and -1 , then the spectrum of the self-adjoint Hankel operator $H(\omega)$ is asymptotically symmetric with respect to the reflection $\lambda \mapsto-\lambda$. For compact operators $\Gamma(h)$ with such symbols, this means that the leading terms of the asymptotics of $\lambda^{+}(\Gamma(h))$ and $\lambda^{-}(\Gamma(h))$ coincide.

For our purposes, it suffices to consider the case when sing supp $\omega$ consists of a finite number of points.

1.5. The structure of the paper. Along with the representation in the space $\ell^{2}\left(\mathbb{Z}_{+}\right)$, Hankel operators can be defined as integral operators $\Gamma(\mathbf{h})$ in $L^{2}\left(\mathbb{R}_{+}\right)$with kernels $\mathbf{h}(t+s)$. We will refer to the Hankel operators $\Gamma(h)$ acting in $\ell^{2}\left(\mathbb{Z}_{+}\right)$as to the discrete representation, and to the Hankel operators $\Gamma(\mathbf{h})$ acting in $L^{2}\left(\mathbb{R}_{+}\right)$as to the continuous representation. Similarly to the realization of operators $\Gamma(h)$ in the Hardy space $H^{2}(\mathbb{T})$ described in Section 1.1, "continuous" Hankel operators $\Gamma(\mathbf{h})$ can be realized in the Hardy space $H^{2}(\mathbb{R})$ of functions analytic in the upper half-plane. We use boldface font for objects associated with the continuous representation. We have tried to make exposition in the discrete and continuous cases parallel as much as possible.

We collect necessary operator theoretic background in Section 2; the key results of that section are Theorems 2.3 and 2.7. In Section 3 we prove the localization 
principle, see Theorems 3.2 and 3.3 (one of these theorems refers to the discrete representation and another one to the continuous one). In Section 4 we prove the symmetry principle, see Theorems 4.1 and 4.3 (again, those are the discrete and the continuous versions).

Applications of these general results to Hankel operators $\Gamma(h)$ and $\Gamma(\mathbf{h})$ are given in Sections 5 and 6, respectively. The main result for the operators $\Gamma(h)$ is stated as Theorem 5.7. Here we prove formulas (1.8), (1.9) for sequences $h(j)$ with asymptotics (1.6) as $j \rightarrow \infty$. Moreover, in Section 5.4 we discuss the version of Theorem 5.7 for Hankel operators acting on the Hardy space $H^{2}(\mathbb{T})$.

The results on the asymptotic behavior of eigenvalues of integral Hankel operators $\boldsymbol{\Gamma}(\mathbf{h})$ are stated similarly to the discrete case. However, we have to take into account the fact that $\mathbf{h}(t)$ may be singular both as $t \rightarrow \infty$ and as $t \rightarrow 0$. Note that oscillating terms appear for $t \rightarrow \infty$ only. Singularities of $\mathbf{h}(t)$ at points $t_{0}>0$ are also not excluded.

\section{Abstract operator theoretic input}

The main results of this section are Theorems 2.3 and 2.7. They are used for the proofs of the localization principle in Section 3 and of the symmetry principle in Section 4.

2.1. Notation. Here we recall some notation related to eigenvalues and singular values of compact operators. For a compact self-adjoint operator $A$, we denote by $\left\{\lambda_{n}^{+}(A)\right\}_{n=1}^{\infty}$ the non-increasing sequence of positive eigenvalues of $A$; we assume that the eigenvalues are enumerated with multiplicities and that the sequence $\left\{\lambda_{n}^{+}(A)\right\}_{n=1}^{\infty}$ is appended by zeros if $A$ has only finitely many positive eigenvalues. We also set $\lambda_{n}^{-}(A)=\lambda_{n}^{+}(-A)$. The singular values of a (not necessarily self-adjoint) compact operator $A$ are defined by $s_{n}(A)=\lambda_{n}^{+}(|A|)$ where $|A|=\sqrt{A^{*} A}$.

To describe the power asymptotics of the type (1.8) and (1.12), it is convenient to define the following functionals. For $p>0$, and for a compact operator $A$, we set

$$
\Delta_{p}(A)=\limsup _{n \rightarrow \infty} n s_{n}(A)^{p}, \quad \delta_{p}(A)=\liminf _{n \rightarrow \infty} n s_{n}(A)^{p} .
$$

Moreover, if $A$ is self-adjoint, we denote

$$
\Delta_{p}^{ \pm}(A)=\limsup _{n \rightarrow \infty} n \lambda_{n}^{ \pm}(A)^{p}, \quad \delta_{p}^{ \pm}(A)=\liminf _{n \rightarrow \infty} n \lambda_{n}^{ \pm}(A)^{p} .
$$

Put $A_{ \pm}=(|A| \pm A) / 2$ so that $A_{ \pm}= \pm \mathbb{1}_{\mathbb{R}_{ \pm}}(A) A$. Since $\lambda_{n}^{ \pm}(A)=s_{n}\left(A_{ \pm}\right)$for an arbitrary self-adjoint operator $A$, we have

$$
\Delta_{p}^{ \pm}(A)=\Delta_{p}\left(A_{ \pm}\right), \quad \delta_{p}^{ \pm}(A)=\delta_{p}\left(A_{ \pm}\right)
$$

In all concrete applications, our upper limits will coincide with the lower limits; however, we work with the upper and lower limits separately because it is more general and, at the same time, it is technically more convenient. 
We denote by $\mathbf{S}_{p, \infty}$ the class of all compact operators $A$ such that $\Delta_{p}(A)$ is finite, and by $\mathbf{S}_{p, \infty}^{0} \subset \mathbf{S}_{p, \infty}$ the subclass of all operators $A$ such that $\Delta_{p}(A)=0$. It is well known that both $\mathbf{S}_{p, \infty}$ and $\mathbf{S}_{p, \infty}^{0}$ are ideals of the algebra of bounded operators $\mathcal{B}$; in particular, they are linear spaces. Of course $A \in \mathbf{S}_{p, \infty}\left(\right.$ or $A \in \mathbf{S}_{p, \infty}^{0}$ ) if and only if the same is true for its adjoint $A^{*}$. We set $\mathbf{S}_{0}=\cap_{p>0} \mathbf{S}_{p, \infty}$, that is,

$$
A \in \mathbf{S}_{0} \quad \Leftrightarrow \quad s_{n}(A)=O\left(n^{-\alpha}\right), \quad n \rightarrow \infty, \quad \forall \alpha>0 .
$$

It is convenient to make use of the counting functions

$$
n(\lambda ; A)=\#\left\{n: s_{n}(A)>\lambda\right\}, \quad n_{ \pm}(\lambda ; A)=\#\left\{n: \lambda_{n}^{ \pm}(A)>\lambda\right\}, \quad \lambda>0 .
$$

In terms of these functions, we have

$$
\Delta_{p}(A)=\limsup _{\lambda \rightarrow 0} \lambda^{p} n(\lambda ; A), \quad \Delta_{p}^{ \pm}(A)=\limsup _{\lambda \rightarrow 0} \lambda^{p} n_{ \pm}(\lambda ; A),
$$

and similarly for the lower limits.

Asymptotic formulas for singular values and eigenvalues can be equivalently rewritten in terms of the functionals (2.1) and (2.2). We make a standing assumption that the indices $\alpha>0$ and $p>0$ are related by $p=1 / \alpha$. Then

$$
\lim _{n \rightarrow \infty} n^{\alpha} s_{n}(A)=c \Longleftrightarrow \Delta_{p}(A)=\delta_{p}(A)=c^{p}
$$

and

$$
\lim _{n \rightarrow \infty} n^{\alpha} \lambda_{n}^{ \pm}(A)=c^{ \pm} \Longleftrightarrow \Delta_{p}^{ \pm}(A)=\delta_{p}^{ \pm}(A)=\left(c^{ \pm}\right)^{p} .
$$

Since, for self-adjoint operators $A$, the sequence $s_{n}(A)$ is the union of the two sequences $\lambda_{n}^{+}(A)$ and $\lambda_{n}^{-}(A)$, for the counting functions we have

$$
n(\lambda ; A)=n_{+}(\lambda ; A)+n_{-}(\lambda ; A), \quad \lambda>0 .
$$

2.2. Asymptotically orthogonal operators. First we recall a lemma which goes back to $\mathrm{H}$. Weyl.

Lemma 2.1. [2, Section 11.6] Let $A$ be a compact operator and let $B \in \mathbf{S}_{p, \infty}^{0}$ for some $p>0$. Then

$$
\Delta_{p}(A+B)=\Delta_{p}(A) \quad \text { and } \quad \delta_{p}(A+B)=\delta_{p}(A) .
$$

If $A$ and $B$ are self-adjoint, then also

$$
\Delta_{p}^{ \pm}(A+B)=\Delta_{p}^{ \pm}(A) \quad \text { and } \quad \delta_{p}^{ \pm}(A+B)=\delta_{p}^{ \pm}(A) .
$$

Recall the implication (see, e.g. [2, Theorem 11.6.9])

$$
A \in \mathbf{S}_{p, \infty}, \quad B \in \mathbf{S}_{p, \infty} \quad \Rightarrow \quad A^{*} B \in \mathbf{S}_{p / 2, \infty}, \quad A B^{*} \in \mathbf{S}_{p / 2, \infty} .
$$

We will say that the operators $A$ and $B$ in $\mathbf{S}_{p, \infty}$ are asymptotically orthogonal if the class $\mathbf{S}_{p / 2, \infty}$ in the right side of (2.8) can be replaced by its subclass $\mathbf{S}_{p / 2, \infty}^{0}$. 
Theorem 2.2. [13, Theorem 2.2] Let $A_{1}, \ldots, A_{L}$ be compact operators such that for some $p>0$

$$
A_{\ell}^{*} A_{j} \in \mathbf{S}_{p / 2, \infty}^{0}, \quad A_{\ell} A_{j}^{*} \in \mathbf{S}_{p / 2, \infty}^{0} \quad \text { for all } \ell \neq j .
$$

Then for $A=A_{1}+\cdots+A_{L}$, we have

$$
\Delta_{p}(A) \leq \sum_{\ell=1}^{L} \Delta_{p}\left(A_{\ell}\right), \quad \delta_{p}(A) \geq \sum_{\ell=1}^{L} \delta_{p}\left(A_{\ell}\right) .
$$

In particular, if $\Delta_{p}\left(A_{\ell}\right)=\delta_{p}\left(A_{\ell}\right)$ for all $\ell$, then

$$
\Delta_{p}\left(A_{\ell}\right)=\delta_{p}\left(A_{\ell}\right)=\sum_{\ell=1}^{L} \Delta_{p}\left(A_{\ell}\right) .
$$

A very similar (only a slightly weaker) result was obtained much earlier in [3]; note that the proofs in [3] and [13] are quite different.

Here we need an analogue of this statement for self-adjoint operators.

Theorem 2.3. Let $A_{1}, \ldots, A_{L}$ be compact self-adjoint operators such that for some $p>0$

$$
A_{\ell} A_{j} \in \mathbf{S}_{p / 2, \infty}^{0} \quad \text { for all } \ell \neq j .
$$

Then for $A=A_{1}+\cdots+A_{L}$, we have

$$
\Delta_{p}^{ \pm}(A) \leq \sum_{\ell=1}^{L} \Delta_{p}^{ \pm}\left(A_{\ell}\right), \quad \delta_{p}^{ \pm}(A) \geq \sum_{\ell=1}^{L} \delta_{p}^{ \pm}\left(A_{\ell}\right) .
$$

In particular, if $\Delta_{p}^{ \pm}\left(A_{\ell}\right)=\delta_{p}^{ \pm}\left(A_{\ell}\right)$ for all $\ell$, then

$$
\Delta_{p}^{ \pm}(A)=\delta_{p}^{ \pm}(A)=\sum_{\ell=1}^{L} \Delta_{p}^{ \pm}\left(A_{\ell}\right)
$$

Again, a version of this theorem can be found in [3]; here we give a different proof. In order to explain the intuition behind this theorem, we observe that the estimates (2.10) are quite obvious if the operators $A_{\ell}$ are orthogonal in the sense that

$$
A_{j} A_{\ell}=0, \quad \forall j \neq \ell
$$

Then $A=A_{1}+\cdots+A_{L}$ is a "block-diagonal" operator acting in the direct sum $\oplus_{\ell=1}^{L} \overline{\operatorname{Ran}\left(A_{\ell}\right)}$. It follows that

$$
n_{ \pm}(\lambda ; A)=\sum_{\ell=1}^{L} n_{ \pm}\left(\lambda ; A_{\ell}\right), \quad \lambda>0 .
$$

Multiplying this by $\lambda^{p}$, taking limsup (resp. liminf) as $\lambda \rightarrow 0$ and recalling the expressions (2.5) for $\Delta_{p}^{ \pm}, \delta_{p}^{ \pm}$in terms of the counting functions, we obtain the first 
(resp. the second) inequality in (2.10). Our goal is to replace the trivial condition (2.11) by a much weaker assumption (2.9).

In order to prove Theorem 2.3, we will need the following auxiliary assertions.

Lemma 2.4. [3, Proposition 4] Let $p>0$, and let $M_{0}, M_{1}$ be bounded non-negative self-adjoint operators such that $M_{1}-M_{0} \in \mathbf{S}_{p / 2, \infty}^{0}$. Then $M_{1}^{1 / 2}-M_{0}^{1 / 2} \in \mathbf{S}_{p, \infty}^{0}$.

The proof of this lemma in [3] uses a non-trivial estimate of [1, Theorem 3]:

$$
\Delta_{p}^{ \pm}\left(M_{1}^{1 / 2}-M_{0}^{1 / 2}\right) \leq c(p)\left(\Delta_{p / 2}^{ \pm}\left(M_{1}-M_{0}\right)\right)^{1 / 2}
$$

Lemma 2.5. Let $A, B$ be self-adjoint operators in $\mathbf{S}_{p, \infty}$ such that $A B \in \mathbf{S}_{p / 2, \infty}^{0}$. Then

$$
|A+B|-(|A|+|B|) \in \mathbf{S}_{p, \infty}^{0}
$$

Proof. Since

$$
|A+B|^{2}-(|A|+|B|)^{2}=A B+B A-|A||B|+|B||A|
$$

and $|A||B|=\operatorname{sign}(A) A B \operatorname{sign}(B)$, expression (2.13) belongs to $\mathbf{S}_{p / 2, \infty}^{0}$. So it remains to apply Lemma 2.4 with $M_{0}=(|A|+|B|)^{2}, M_{1}=|A+B|^{2}$.

Adding and subtracting $A+B$ in (2.12), we obtain

Corollary 2.6. Under the assumptions of Lemma 2.5, the inclusions

$$
(A+B)_{ \pm}-\left(A_{ \pm}+B_{ \pm}\right) \in \mathbf{S}_{p, \infty}^{0}
$$

hold.

2.3. Proof of Theorem 2.3. Using induction in $L$, it is easy to reduce the problem to the case $L=2$. Thus, changing our notation slighly, we will assume that $A, B$ are self-adjoint operators in $\mathbf{S}_{p, \infty}$ and $A B \in \mathbf{S}_{p / 2, \infty}^{0}$. We will prove that

$$
\begin{aligned}
\Delta_{p}^{ \pm}(A+B) & \leq \Delta_{p}^{ \pm}(A)+\Delta_{p}^{ \pm}(B), \\
\delta_{p}^{ \pm}(A+B) & \geq \delta_{p}^{ \pm}(A)+\delta_{p}^{ \pm}(B) .
\end{aligned}
$$

According to the statement (2.7) of Lemma 2.1, it follows from (2.14) that

$$
\Delta_{p}\left((A+B)_{ \pm}\right)=\Delta_{p}\left(A_{ \pm}+B_{ \pm}\right) .
$$

Since $A_{ \pm} B_{ \pm} \in \mathbf{S}_{p / 2, \infty}^{0}$, Theorem 2.2 implies that

$$
\Delta_{p}\left(A_{ \pm}+B_{ \pm}\right) \leq \Delta_{p}\left(A_{ \pm}\right)+\Delta_{p}\left(B_{ \pm}\right)
$$

Combining (2.17), (2.18) and taking (2.3) into account, we conclude the proof of (2.15). The estimate (2.16) for the lower limits can be obtained in a similar way. 
2.4. Symmetry with respect to the reflection around zero. The meaning of the following result is that if a compact self-adjoint operator $A$ is "almost" unitarily equivalent to $-A$, then its positive and negative eigenvalues have the same asymptotic behaviour.

Theorem 2.7. Let $p>0$, and let $A$ be a compact self-adjoint operator such that for some unitary operator $U$, we have

$$
R:=U A U^{*}+A \in \mathbf{S}_{p, \infty}^{0}
$$

Then

$$
\begin{gathered}
\Delta_{p}^{+}(A)=\Delta_{p}^{-}(A)=\frac{1}{2} \Delta_{p}(A), \\
\delta_{p}^{+}(A)=\delta_{p}^{-}(A)=\frac{1}{2} \delta_{p}(A) .
\end{gathered}
$$

Proof. The first equalities in (2.20), (2.21) are easy to check. Indeed, according to Lemma 2.1 it follows from (2.19) that

$$
\begin{aligned}
\Delta_{p}^{+}(A) & =\Delta_{p}^{+}\left(U A U^{*}\right)=\Delta_{p}^{+}(-A)=\Delta_{p}^{-}(A), \\
\delta_{p}^{+}(A) & =\delta_{p}^{+}\left(U A U^{*}\right)=\delta_{p}^{+}(-A)=\delta_{p}^{-}(A) .
\end{aligned}
$$

The second pair of equalities is more delicate. Let us multiply (2.6) by $\lambda^{p}$. Passing to the upper limit as $\lambda \rightarrow 0$ and using the definition (2.5) of the quantities $\Delta_{p}$, we see that

$$
\Delta_{p}(A) \leq \Delta_{p}^{+}(A)+\Delta_{p}^{-}(A)=2 \Delta_{p}^{+}(A) .
$$

Similarly, passing to the lower limit, we see that

$$
\delta_{p}(A) \geq \delta_{p}^{+}(A)+\delta_{p}^{-}(A)=2 \delta_{p}^{+}(A) .
$$

It remains to prove the opposite estimates. In view of (2.6) for all $\lambda>0$, we have

$$
\begin{aligned}
n(\lambda ; A)=n_{+}(\lambda ; A)+n_{-}(\lambda ; A)=n_{+}(\lambda ; A)+n_{-}\left(\lambda ; U A U^{*}\right) \\
\quad=n_{+}(\lambda ; A)+n_{-}(\lambda ;-A+R)=n_{+}(\lambda ; A)+n_{+}(\lambda ; A-R) .
\end{aligned}
$$

For any compact self-adjoint $A_{1}, A_{2}$ we have the inequality (see e.g. [2, Theorem $9.2 .9])$

$$
n_{+}\left(\lambda_{1}+\lambda_{2} ; A_{1}+A_{2}\right) \leq n_{+}\left(\lambda_{1} ; A_{1}\right)+n_{+}\left(\lambda_{2} ; A_{2}\right), \quad \lambda_{1}, \lambda_{2}>0 .
$$

In particular, for every $\varepsilon \in(0,1)$

$$
n_{+}((1+\varepsilon) \lambda ; A) \leq n_{+}(\lambda ; A-R)+n_{+}(\varepsilon \lambda ; R)
$$

and therefore (2.23) yields the estimate $n(\lambda ; A) \geq n_{+}(\lambda ; A)+n_{+}((1+\varepsilon) \lambda ; A)-n_{+}(\varepsilon \lambda ; R) \geq 2 n_{+}((1+\varepsilon) \lambda ; A)-n_{+}(\varepsilon \lambda ; R)$. 
Let us multiply this estimate by $\lambda^{p}$ and pass to the upper limit as $\lambda \rightarrow 0$. Since $R \in \mathbf{S}_{p, \infty}^{0}$, we have $\lambda^{p} n_{+}(\varepsilon \lambda ; R) \rightarrow 0$. Therefore according to definition (2.5), we find that

$$
\Delta_{p}(A) \geq 2(1+\varepsilon)^{-p} \Delta_{p}^{+}(A) .
$$

Hence $\Delta_{p}(A) \geq 2 \Delta_{p}^{+}(A)$ because $\varepsilon>0$ is arbitrary. Together with (2.22), this proves $(2.20)$.

Similarly, (2.24) implies that for every $\varepsilon \in(0,1)$

$$
n_{+}(\lambda ; A-R) \leq n_{+}((1-\varepsilon) \lambda ; A)+n_{+}(\varepsilon \lambda ;-R)
$$

and therefore (2.23) yields the estimate

$n(\lambda ; A) \leq n_{+}(\lambda ; A)+n_{+}((1-\varepsilon) \lambda ; A)+n_{+}(\varepsilon \lambda ;-R) \leq 2 n_{+}((1-\varepsilon) \lambda ; A)+n_{+}(\varepsilon \lambda ;-R)$.

Let us again multiply it by $\lambda^{p}$ and then pass to the lower limit whence

$$
\delta_{p}(A) \leq 2(1-\varepsilon)^{-p} \delta_{p}^{+}(A) .
$$

This suffices to conclude the proof of (2.21).

\section{LOCALIZATION PRINCIPLE}

The localization principle for eigenvalues of Hankel operators in the spaces $H^{2}(\mathbb{T})$ and $H^{2}(\mathbb{R})$ will be stated in Theorems 3.2 and 3.3 , respectively.

3.1. Hankel operators in $H^{2}(\mathbb{T})$. In a standard way, we define the singular support sing supp $\omega$ of a function $\omega \in L^{\infty}(\mathbb{T})$ as the smallest closed set $X \subset \mathbb{T}$ such that $\omega \in C^{\infty}(\mathbb{T} \backslash X)$. Recall also that the class $\mathbf{S}_{0}$ of compact operators was defined by (2.4).

The key analytic ingredient of the proof of Therem 3.2 is the following statement.

Lemma 3.1. [13, Lemma 2.6] Let $\omega_{1}, \omega_{2} \in L^{\infty}(\mathbb{T})$ be such that sing supp $\omega_{1} \cap$ sing $\operatorname{supp} \omega_{2}=\varnothing$. Then

$$
H\left(\omega_{1}\right)^{*} H\left(\omega_{2}\right) \in \mathbf{S}_{0} .
$$

Below we state the localization principle for eigenvalues of self-adjoint Hankel operators. We use the functionals $\Delta_{p}^{ \pm}$and $\delta_{p}^{ \pm}$, defined by formulas (2.2).

Theorem 3.2. Let $\omega_{1}, \ldots, \omega_{L} \in L^{\infty}(\mathbb{T})$ be symbols such that the singular supports of $\omega_{\ell}$ are disjoint:

$$
\operatorname{sing} \operatorname{supp} \omega_{\ell} \cap \operatorname{sing} \operatorname{supp} \omega_{j}=\varnothing, \quad \ell \neq j,
$$

and such that the symmetry condition (1.2) is satisfied. Then for $\omega=\omega_{1}+\cdots+\omega_{L}$ and for an arbitrary $p>0$, we have

$$
\Delta_{p}^{ \pm}(H(\omega)) \leq \sum_{\ell=1}^{L} \Delta_{p}^{ \pm}\left(H\left(\omega_{\ell}\right)\right), \quad \delta_{p}^{ \pm}(H(\omega)) \geq \sum_{\ell=1}^{L} \delta_{p}^{ \pm}\left(H\left(\omega_{\ell}\right)\right) .
$$


In particular, if $\Delta_{p}^{ \pm}\left(H\left(\omega_{\ell}\right)\right)=\delta_{p}^{ \pm}\left(H\left(\omega_{\ell}\right)\right)$ for all $\ell$, then

$$
\Delta_{p}^{ \pm}(H(\omega))=\delta_{p}^{ \pm}(H(\omega))=\sum_{\ell=1}^{L} \Delta_{p}^{ \pm}\left(H\left(\omega_{\ell}\right)\right) .
$$

Proof. It suffices to use Theorem 2.3 with $A_{\ell}=H\left(\omega_{\ell}\right)$. The inclusion $A_{\ell} A_{j} \in \mathbf{S}_{p / 2, \infty}^{0}$ for $\ell \neq j$ follows from Lemma 3.1.

Note that the expressions in (3.1) may be infinite. In [13], we have an exact analogue of Theorem 2.3 for singular values of (not necessarily self-adjoint) Hankel operators.

3.2. Hankel operators in $H^{2}(\mathbb{R})$. Hankel operators can also be defined in the Hardy space $H^{2}(\mathbb{R})$ of functions analytic in the upper half-plane. We denote by $\Phi f=\widehat{f}$ the Fourier transform of $f$ in $L^{2}(\mathbb{R})$,

$$
(\Phi f)(t)=\widehat{f}(t)=\frac{1}{\sqrt{2 \pi}} \int_{-\infty}^{\infty} f(x) e^{-i x t} d x
$$

Let $H^{2}(\mathbb{R}) \subset L^{2}(\mathbb{R})$ be the Hardy class,

$$
H^{2}(\mathbb{R})=\left\{f \in L^{2}: \widehat{f}(t)=0 \text { for } t<0\right\},
$$

and let $\mathbf{P}_{+}: L^{2}(\mathbb{R}) \rightarrow H^{2}(\mathbb{R})$ be the corresponding orthogonal projection. Let $\mathbf{W}$ be the involution in $L^{2}(\mathbb{R}),(\mathbf{W} f)(x)=f(-x)$. For $\omega \in L^{\infty}(\mathbb{R})$, the Hankel operator $\mathbf{H}(\boldsymbol{\omega})$ in $H^{2}(\mathbb{R})$ is defined by

$$
\mathbf{H}(\boldsymbol{\omega}) f=\mathbf{P}_{+}(\boldsymbol{\omega} \mathbf{W} f), \quad f \in H^{2}(\mathbb{R}) .
$$

It is straightforward to see that the symmetry condition

$$
\overline{\omega(x)}=\omega(-x), \quad x \in \mathbb{R},
$$

ensures that $\mathbf{H}(\boldsymbol{\omega})$ is self-adjoint.

There is a unitary equivalence between the Hankel operators $H(\omega)$ defined in $H^{2}(\mathbb{T})$ by formula (1.1) and the Hankel operators $\mathbf{H}(\boldsymbol{\omega})$ defined in $H^{2}(\mathbb{R})$ by formula (3.3). Indeed, let

$$
w=\frac{z-i / 2}{z+i / 2}, \quad z=\frac{i}{2} \frac{1+w}{1-w},
$$

be the standard conformal map sending the upper half-plane onto the unit disc, and let $\mathcal{U}: L^{2}(\mathbb{T}) \rightarrow L^{2}(\mathbb{R})$ be the corresponding unitary operator defined by

$$
(\mathcal{U} f)(x)=\frac{1}{\sqrt{2 \pi}} \frac{1}{x+i / 2} f\left(\frac{x-i / 2}{x+i / 2}\right), \quad\left(\mathcal{U}^{*} \mathbf{f}\right)(\mu)=i \sqrt{2 \pi} \frac{1}{1-\mu} \mathbf{f}\left(\frac{i}{2} \frac{1+\mu}{1-\mu}\right) .
$$

Then

provided

$$
\mathcal{U} H(\omega) \mathcal{U}^{*}=\mathbf{H}(\omega)
$$

$$
\omega(x)=-\frac{x-i / 2}{x+i / 2} \omega\left(\frac{x-i / 2}{x+i / 2}\right) .
$$


Symbols $\omega(x)$ of Hankel operators (3.3) have the exceptional point $x=\infty$. In order to rewrite the results obtained for Hankel operators $H(\omega)$ in terms of the Hankel operators $\mathbf{H}(\boldsymbol{\omega})$, we identify the points $x=+\infty$ and $x=-\infty$. The real line with such identification will be denoted $\mathbb{R}_{*}$. We write $\boldsymbol{\omega} \in C\left(\mathbb{R}_{*}\right)$ if $\boldsymbol{\omega} \in C(\mathbb{R})$ and if

$$
\lim _{x \rightarrow \infty} \boldsymbol{\omega}(x)=\lim _{x \rightarrow-\infty} \boldsymbol{\omega}(x) .
$$

Similarly, we write $\boldsymbol{\omega} \in C^{\infty}\left(\mathbb{R}_{*}\right)$ if $\boldsymbol{\omega} \in C^{\infty}(\mathbb{R})$ and

$$
\lim _{x \rightarrow \infty} \boldsymbol{\omega}^{(m)}(x)=\lim _{x \rightarrow-\infty} \boldsymbol{\omega}^{(m)}(x) .
$$

In particular, the point $x=\infty$ belongs to the singular support of $\boldsymbol{\omega}(x)$ if for some $m \geq 0$ the relation (3.7) fails (i.e. if either at least one of the limits does not exist or if the limits are not equal).

In view of relations (3.5) and (3.6) the localization principle for Hankel operators in $H^{2}(\mathbb{R})$ given below is a direct consequence of the localization principle in $H^{2}(\mathbb{T})$ (Theorem 3.2).

Theorem 3.3. Let $\boldsymbol{\omega}_{1}, \ldots, \boldsymbol{\omega}_{L} \in C\left(\mathbb{R}_{*}\right)$ be symbols satisfying the symmetry condition

$$
\overline{\omega(x)}=\omega(-x)
$$

and such that the singular supports of $\boldsymbol{\omega}_{\ell}$ for different $\ell$ are disjoint. Then for the symbol $\boldsymbol{\omega}=\omega_{1}+\cdots \omega_{L}$ and for any $p>0$ we have

$$
\Delta_{p}^{ \pm}(\mathbf{H}(\boldsymbol{\omega})) \leq \sum_{\ell=1}^{L} \Delta_{p}^{ \pm}\left(\mathbf{H}\left(\boldsymbol{\omega}_{\ell}\right)\right), \quad \delta_{p}^{ \pm}(\mathbf{H}(\omega)) \geq \sum_{\ell=1}^{L} \delta_{p}^{ \pm}\left(\mathbf{H}\left(\boldsymbol{\omega}_{\ell}\right)\right) .
$$

In particular, if $\Delta_{p}^{ \pm}\left(\mathbf{H}\left(\boldsymbol{\omega}_{\ell}\right)\right)=\delta_{p}^{ \pm}\left(\mathbf{H}\left(\boldsymbol{\omega}_{\ell}\right)\right)$ for all $\ell$, then

$$
\Delta_{p}^{ \pm}(\mathbf{H}(\boldsymbol{\omega}))=\delta_{p}^{ \pm}(\mathbf{H}(\boldsymbol{\omega}))=\sum_{\ell=1}^{L} \Delta_{p}^{ \pm}\left(\mathbf{H}\left(\boldsymbol{\omega}_{\ell}\right)\right) .
$$

\section{SymmetRY PRINCIPLE}

The symmetry principle for the eigenvalues of Hankel operators in the spaces $H^{2}(\mathbb{T})$ and $H^{2}(\mathbb{R})$ will be stated in Theorems 4.1 and 4.3, respectively, in terms of the functionals $\Delta_{p}^{ \pm}, \delta_{p}^{ \pm}$(see (2.1) and (2.2) $)$. Moreover, in Section 4.3 we discuss the symmetry principle for the essential spectrum of Hankel operators.

4.1. Symmetry principle in $H^{2}(\mathbb{T})$. The symmetry principle for compact selfadjoint Hankel operators $H(\omega)$ in the space $L^{2}(\mathbb{T})$ can be stated as follows. 
Theorem 4.1. Let $\omega \in L^{\infty}(\mathbb{T})$ be a symbol satisfying the symmetry condition (1.2) and such that $\operatorname{sing} \operatorname{supp} \omega$ does not contain the points 1 and -1 . Then for any $p>0$,

$$
\begin{aligned}
\Delta_{p}^{+}(H(\omega)) & =\Delta_{p}^{-}(H(\omega))=\frac{1}{2} \Delta_{p}(H(\omega)), \\
\delta_{p}^{+}(H(\omega)) & =\delta_{p}^{-}(H(\omega))=\frac{1}{2} \delta_{p}(H(\omega)) .
\end{aligned}
$$

If $\operatorname{sing} \operatorname{supp} \omega$ contains the points 1 or -1 , the symmetry breaks down: the contribution of each of these points to the spectrum is not symmetric. This will be illustrated below by Theorem 5.10 .

Put

$$
s(\mu)=\operatorname{sign} \operatorname{Im} \mu, \quad \mu \in \mathbb{T} .
$$

The operator of multiplication by $s$ in $L^{2}(\mathbb{T})$ will also be denoted by $s$. Clearly, $s=s^{*}$ and $s^{2}=I$. In the following statement, $H(\omega)$ is not necessarily self-adjoint.

Below it will be convenient to consider Hankel operators $H(\omega)$ as operators acting not on the Hardy class, but on the space $L^{2}(\mathbb{T})$; in this case $H(\omega)$ is defined by the formula

$$
H(\omega)=P_{+} \omega W P_{+} .
$$

Of course, the non-zero spectra of the operators (1.1) and (4.2) coincide.

Lemma 4.2. Let $\omega \in L^{\infty}(\mathbb{T})$ be such that the singular support of $\omega$ does not contain the points 1 and -1 . Then

$$
s H(\omega)+H(\omega) s \in \mathbf{S}_{0} .
$$

Proof. We will use two well-known facts (see the book [8] and Lemma 4.2 in [13], for additional details):

(i) if $\sigma \in C^{\infty}(\mathbb{T})$, then $H(\sigma) \in \mathbf{S}_{0}$;

(ii) if $\sigma \in C^{\infty}(\mathbb{T})$, then the commutator $\left[\sigma, P_{+}\right]:=\sigma P_{+}-P_{+} \sigma \in \mathbf{S}_{0}$.

Write $\omega=\omega_{0}+\omega_{1}$, where $\omega_{0} \in L^{\infty}(\mathbb{T})$ vanishes identically in a neighborhood of $\{-1,1\}$, and $\omega_{1} \in C^{\infty}(\mathbb{T})$. By (i), it suffices to prove (4.3) with $\omega_{0}$ instead of $\omega$. In what follows, we drop the subscript 0 and simply assume that $\omega$ vanishes in a neighborhood of $\{-1,1\}$.

Put $\omega_{*}(\mu)=\omega(\bar{\mu})$. Let us choose $\varphi \in C^{\infty}(\mathbb{T})$ such that $\varphi \omega=\omega$ and $\varphi \omega_{*}=\omega_{*}$, and $\varphi$ vanishes in a neighborhood of $\{-1,1\}$. Then we also have $s \varphi \in C^{\infty}(\mathbb{T})$. It follows from (ii) that

$$
\left[s, P_{+}\right] \varphi=s P_{+} \varphi-P_{+} s \varphi=s\left[P_{+}, \varphi\right]+\left[s \varphi, P_{+}\right] \in \mathbf{S}_{0},
$$

whence

$$
s P_{+} \omega-P_{+} s \omega=\left[s, P_{+}\right] \omega=\left[s, P_{+}\right] \varphi \omega \in \mathbf{S}_{0}
$$

and, multiplying by $W P_{+}$on the right,

$$
s H(\omega)-P_{+} s \omega W P_{+} \in \mathbf{S}_{0} .
$$

Similarly to (4.4), we have

$$
\omega_{*} P_{+} s-\omega_{*} s P_{+} \in \mathbf{S}_{0} .
$$


Therefore using that $H(\omega)=P_{+} W \omega_{*} P_{+}$and multiplying by $P_{+} W$ on the left, we obtain

$$
H(\omega) s-P_{+} W \omega_{*} s P_{+} \in \mathbf{S}_{0} .
$$

Putting together (4.5), (4.6) and taking into account that $s \omega W+W \omega_{*} s=0$, we conclude the proof of (4.3).

Proof of Theorem 4.1. It remains to use Theorem 2.7 with $A=H(\omega)$ and $U=s$; the inclusion (2.19) in the hypothesis of this theorem holds true by Lemma 4.2.

4.2. Symmetry principle in $H^{2}(\mathbb{R})$. The symmetry principles in $H^{2}(\mathbb{T})$ and $H^{2}(\mathbb{R})$ are equivalent.

Theorem 4.3. Let $\boldsymbol{\omega} \in L^{\infty}(\mathbb{R})$ satisfy the symmetry relation $\overline{\boldsymbol{\omega}(x)}=\boldsymbol{\omega}(-x)$, and suppose that sing supp $\boldsymbol{\omega}$ does not contain 0 or $\infty$. Then for any $p>0$,

$$
\begin{aligned}
\Delta_{p}^{+}(\mathbf{H}(\boldsymbol{\omega})) & =\Delta_{p}^{-}(\mathbf{H}(\boldsymbol{\omega}))=\frac{1}{2} \Delta_{p}(\mathbf{H}(\boldsymbol{\omega})), \\
\delta_{p}^{+}(\mathbf{H}(\boldsymbol{\omega})) & =\delta_{p}^{-}(\mathbf{H}(\boldsymbol{\omega}))=\frac{1}{2} \delta_{p}(\mathbf{H}(\boldsymbol{\omega})) .
\end{aligned}
$$

Proof. Observe that the map (3.4) sends the point $z=\infty$ into the point $w=1$ and the point $z=0$ into the point $w=-1$. Let the symbol $\omega$ be defined by formula (3.6). Since its singular support does not contain the points 1 and -1 , Theorem 4.1 applies to the Hankel operator $H(\omega)$. According to (3.5) the operators $H(\omega)$ and $\mathbf{H}(\omega)$ are unitarily equivalent, which yields (4.7) and (4.8).

4.3. Essential spectrum. Although this is not the focus of the present paper, we mention that some variants of the symmetry principle also hold true for noncompact Hankel operators. For example, we have

Theorem 4.4. Let $\omega \in L^{\infty}(\mathbb{T})$ be a symbol satisfying the symmetry condition (1.2). Suppose that $\omega$ is continuous in some neighborhoods of the points 1 and -1 . Then

$$
\sigma_{\text {ess }}(H(\omega))=\sigma_{\text {ess }}(-H(\omega)) .
$$

Proof. Let $\omega$ be continuous on the union $G$ of two arcs $\left[e^{-i \delta}, e^{i \delta}\right]$ and $\left[-e^{i \delta},-e^{-i \delta}\right]$ for some $\delta>0$. There exist functions $\omega_{n} \in C^{\infty}(G)$ such that $\left\|\omega-\omega_{n}\right\|_{L^{\infty}(G)} \rightarrow 0$ as $n \rightarrow \infty$. We set $\omega_{n}(\mu)=\omega(\mu)$ for $\mu \in \mathbb{T} \backslash G$. Then $\left\|\omega-\omega_{n}\right\|_{L^{\infty}(\mathbb{T})} \rightarrow 0$ as $n \rightarrow \infty$. It follows that $\left\|H(\omega)-H\left(\omega_{n}\right)\right\| \rightarrow 0$ and hence

$$
\left\|(s H(\omega) s+H(\omega))-\left(s H\left(\omega_{n}\right) s+H\left(\omega_{n}\right)\right)\right\| \rightarrow 0, \quad n \rightarrow \infty,
$$

where $s$ is defined by formula (4.1). By Lemma 4.2, the operators $s H\left(\omega_{n}\right) s+H\left(\omega_{n}\right)$ are compact for all $n$ and so the operator $s H(\omega) s+H(\omega)$ is also compact. Applying H. Weyl's theorem on the stability of the essential spectrum under compact perturbations, we obtain that

$$
\sigma_{\text {ess }}(H(\omega))=\sigma_{\text {ess }}(s H(\omega) s)=\sigma_{\text {ess }}(-H(\omega)),
$$

as required. 
We are not aware of this statement appearing explicitly in the literature, although similar considerations have been used by S. Power in his work [9].

If $\omega$ is discontinuous at 1 or -1 , then in general the symmetry (4.9) breaks down (see formula (1.7)).

Of course Theorem 4.4 can be reformulated in terms of Hankel operators in the space $H^{2}(\mathbb{R})$.

Theorem 4.5. Let $\boldsymbol{\omega} \in L^{\infty}(\mathbb{R})$ be a symbol satisfying the condition (3.8). Suppose that $\boldsymbol{\omega}$ is continuous in neighborhoods of the points 0 and $\infty$. Then

$$
\sigma_{e s s}(\mathbf{H}(\boldsymbol{\omega}))=\sigma_{\text {ess }}(-\mathbf{H}(\boldsymbol{\omega})) .
$$

\section{Spectral ASYmptotics FOR HANKel Operators in $\ell^{2}\left(\mathbb{Z}_{+}\right)$}

Recall that Hankel operators $\Gamma(h)$ in $\ell^{2}\left(\mathbb{Z}_{+}\right)$were defined by formula (1.4). The main result of this section is Theorem [5.7. It gives the asymptotics of eigenvalues of operators $\Gamma(h)$ corresponding to "oscillating" sequences $h$ of the form (1.6). An equivalent result for Hankel operators $H(\omega)$ in the Hardy space $H^{2}(\mathbb{T})$ is stated in Theorem 5.10.

5.1. Previous results. We proceed from a particular case of Theorem 5.7 when the asymptotics of $h(j)$ consists of one term only.

Theorem 5.1. [12, Theorem 1.1] Let $\alpha>0$ and let

$$
q(j)=j^{-1}(\log j)^{-\alpha}, \quad j \geq 2,
$$

(the choice of any finite number of terms of the sequence $q$ is not important). Then the eigenvalues of the Hankel operator $\Gamma(q)$ satisfy the asymptotic relation

$$
\lambda_{n}^{+}(\Gamma(q))=\varkappa(\alpha) n^{-\alpha}+o\left(n^{-\alpha}\right), \quad \lambda_{n}^{-}(\Gamma(q))=o\left(n^{-\alpha}\right),
$$

where the coefficient $\varkappa(\alpha)$ is given by formula (1.10).

Let $q_{-1}(j)=(-1)^{j} q(j)$, and let the unitary operator $T$ in $\ell^{2}\left(\mathbb{Z}_{+}\right)$be defined by the relation $(T u)(j)=(-1)^{j} u(j)$. Then $\Gamma\left(q_{-1}\right)=T^{*} \Gamma(q) T$ whence $\lambda_{n}^{ \pm}\left(\Gamma\left(q_{-1}\right)\right)=$ $\lambda_{n}^{ \pm}(\Gamma(q))$. Therefore Theorem 5.1 yields

Corollary 5.2. The conclusions of Theorem 5.1 are true for the sequence

$$
q_{-1}(j)=(-1)^{j} j^{-1}(\log j)^{-\alpha} .
$$

The first step in the proof of Theorem 5.7 is to construct a symbol corresponding to the sequence (5.1). It is convenient to consider a slightly more general case.

Lemma 5.3. [13, Lemma 4.3] Let $\zeta \in \mathbb{T}, \alpha \geq 0$, and let

$$
q_{\zeta}(j)=\zeta^{-j} j^{-1}(\log j)^{-\alpha}, \quad j \geq 2,
$$


$\left(q_{\zeta}(0)=q_{\zeta}(1)=0\right)$. Put

$$
\omega_{\zeta}(\mu)=\sum_{j=2}^{\infty} j^{-1}(\log j)^{-\alpha}\left((\mu / \zeta)^{j}-(\mu / \zeta)^{-j}\right), \quad \mu \in \mathbb{T} .
$$

Then $\omega_{\zeta} \in L^{\infty}(\mathbb{T})$ and $\omega_{\zeta} \in C^{\infty}(\mathbb{T} \backslash\{\zeta\})$. For the Fourier coefficients of function (5.3), we have $\widehat{\omega}_{\zeta}(j)=q_{\zeta}(j)$ for all $j \geq 0$.

The assertion below is a particular case of our general result (Theorem 3.1 in 13) on the asymptotics of singular values of Hankel operators, needed in the present text. Its proof in [13] uses the localization principle for singular values (which is the analogue of Theorem 3.2).

Theorem 5.4. [13, Theorem 3.1] Let $\alpha>0$, let $\zeta \in \mathbb{T}, \operatorname{Im} \zeta \neq 0$, and let $b \in \mathbb{C}$ be arbitrary. Consider the sequence $h$ given by $h(0)=h(1)=0$ and

$$
h(j)=2 \operatorname{Re}\left(b \zeta^{-j}\right) j^{-1}(\log j)^{-\alpha}, \quad j \geq 2 .
$$

Then the singular values of $\Gamma(h)$ satisfy the asymptotic relation

$$
s_{n}(\Gamma(h))=2^{\alpha} \varkappa(\alpha)|b| n^{-\alpha}+o\left(n^{-\alpha}\right) .
$$

In view of the symmetry principle, this results yields the asymptotics of the eigenvalues of $\Gamma(h)$.

Theorem 5.5. Let $\Gamma(h)$ be the same as in Theorem 5.4. Then

$$
\lambda_{n}^{ \pm}(\Gamma(h))=\varkappa(\alpha)|b| n^{-\alpha}+o\left(n^{-\alpha}\right) .
$$

Proof. Let the symbol $\omega_{\zeta}$ be defined by formula (5.3) and let $\omega=b \omega_{\zeta}+\bar{b} \omega_{\bar{\zeta}}$. Then $\widehat{\omega}(j)=h(j)$ for $j \geq 0$, and hence the operators $\Gamma(h)$ and $H(\omega)$ are unitarily equivalent. By Lemma 5.3, the singular support of $\omega$ consists of the pair of points $\zeta, \bar{\zeta}$. Therefore by the symmetry principle (Theorem 4.1) we have

$$
\Delta_{p}^{+}(\Gamma(h))=\Delta_{p}^{-}(\Gamma(h))=\frac{1}{2} \Delta_{p}(\Gamma(h)),
$$

and similarly for the lower limits. The asymptotic relation (5.4) for the singular values can be equivalently rewritten as $\Delta_{p}(\Gamma(h))=2 \varkappa(\alpha)^{p}|b|^{p}$, and thus we obtain

$$
\Delta_{p}^{+}(\Gamma(h))=\Delta_{p}^{-}(\Gamma(h))=\varkappa(\alpha)^{p}|b|^{p}
$$

and similarly for the lower limits. This yields (5.5).

In order to estimate the error terms, we use the following result of [11]. Let $[\alpha]$ be the integer part of $\alpha,[\alpha]=\max \{m \in \mathbb{Z}: m \leq \alpha\}$. We set

$$
M(\alpha)= \begin{cases}{[\alpha]+1,} & \text { if } \alpha \geq 1 / 2, \\ 0, & \text { if } \alpha<1 / 2 .\end{cases}
$$


For a sequence $g=\{g(j)\}_{j=0}^{\infty}$, we define iteratively the sequences $g^{(m)}=$ $\left\{g^{(m)}(j)\right\}_{j=0}^{\infty}, m=0,1,2, \ldots$, by setting $g^{(0)}(j)=g(j)$ for all $j$ and

$$
g^{(m+1)}(j)=g^{(m)}(j+1)-g^{(m)}(j), \quad j \geq 0 .
$$

Before stating the next result, let us comment that for the sequence $q$ defined by (5.1), the sequences $q^{(m)}$ for all $m \geq 1$ satisfy

$$
q^{(m)}(j)=O\left(j^{-1-m}(\log j)^{-\alpha}\right), \quad j \rightarrow \infty .
$$

On the other hand, the sequence (5.2) with $\zeta \neq 1$ satisfies only the condition $q_{\zeta}^{(m)}(j)=O\left(j^{-1}(\log j)^{-\alpha}\right)$ for any $m \geq 1$. Nevertheless we have the following assertion.

Theorem 5.6. [11, Theorem 2.3] Let $\alpha>0$ and let $M=M(\alpha)$ be the integer given by (5.6). Let $g$ be a complex valued sequence such that

$$
g^{(m)}(j)=o\left(j^{-1-m}(\log j)^{-\alpha}\right), \quad j \rightarrow \infty,
$$

for all $m=0, \ldots, M$. Pick any $\zeta \in \mathbb{T}$ and put $g_{\zeta}(j)=\zeta^{-j} g(j)$. Then $s_{n}\left(\Gamma\left(g_{\zeta}\right)\right)=$ $o\left(n^{-\alpha}\right)$.

5.2. Asymptotics of eigenvalues. Our main result below concerns the real sequences of the form (1.6).

Theorem 5.7. Let $\alpha>0, p=1 / \alpha$; let $\varphi_{1}, \ldots, \varphi_{L} \in(0, \pi)$ be distinct numbers and let $\psi_{1}, \ldots \psi_{L} \in \mathbb{R}$ as well as $\mathrm{b}_{1}, \mathrm{~b}_{-1} \in \mathbb{R}, b_{1}, \ldots, b_{L} \in \mathbb{R}$ be arbitrary. Let $h$ be a sequence of real numbers such that

$$
\begin{aligned}
h(j)=\mathrm{b}_{1} j^{-1}(\log j)^{-\alpha} & +\mathrm{g}_{1}(j)+(-1)^{j}\left(\mathrm{~b}_{-1} j^{-1}(\log j)^{-\alpha}+\mathrm{g}_{-1}(j)\right) \\
& +2 \sum_{\ell=1}^{L}\left(b_{\ell} j^{-1}(\log j)^{-\alpha}+g_{\ell}(j)\right) \cos \left(\varphi_{\ell} j-\psi_{\ell}\right), \quad j \geq 2,
\end{aligned}
$$

where all error terms $\mathrm{g}_{1}, \mathrm{~g}_{-1}, g_{1}, \ldots, g_{L}$ satisfy condition (5.7) for all $m=$ $0,1, \ldots, M(\alpha)(M(\alpha)$ is given by (5.6) $)$. Then the eigenvalues of the Hankel operator $\Gamma(h)$ satisfy the asymptotic relation (1.8) with the coefficients $a^{ \pm}$defined by (1.9).

Proof. It is convenient to give the proof in terms of the functionals $\Delta_{p}^{ \pm}, \delta_{p}^{ \pm}$, (see $(2.2)$ or $(2.5))$. We first consider every term in the right-hand side of (5.8) separately. Put

$$
\mathrm{h}_{1}(j)=\mathrm{b}_{1} j^{-1}(\log j)^{-\alpha}, \quad \mathrm{h}_{-1}(j)=\mathrm{b}_{-1}(-1)^{j} j^{-1}(\log j)^{-\alpha},
$$

and for $\ell=1, \ldots, L$,

$$
h_{\ell}(j)=2 b_{\ell} \cos \left(\varphi_{\ell} j-\psi_{\ell}\right) j^{-1}(\log j)^{-\alpha}=2 \operatorname{Re}\left(b_{\ell} e^{i \psi_{\ell}} \zeta_{\ell}^{-j}\right) j^{-1}(\log j)^{-\alpha},
$$


where $\zeta_{\ell}=e^{i \varphi_{\ell}}$. By Theorem 5.1 and Corollary 5.2, we have

$$
\begin{gathered}
\Delta_{p}^{ \pm}\left(\Gamma\left(\mathrm{h}_{1}\right)\right)=\delta_{p}^{ \pm}\left(\Gamma\left(\mathrm{h}_{1}\right)\right)=\left(\varkappa(\alpha) \mathrm{b}_{1}\right)_{ \pm}^{p}, \\
\Delta_{p}^{ \pm}\left(\Gamma\left(\mathrm{h}_{-1}\right)\right)=\delta_{p}^{ \pm}\left(\Gamma\left(\mathrm{h}_{-1}\right)\right)=\left(\varkappa(\alpha) \mathrm{b}_{-1}\right)_{ \pm}^{p},
\end{gathered}
$$

and by Theorem 5.5, we have

$$
\Delta_{p}^{ \pm}\left(\Gamma\left(h_{\ell}\right)\right)=\delta_{p}^{ \pm}\left(\Gamma\left(h_{\ell}\right)\right)=\left(\varkappa(\alpha)\left|b_{\ell}\right|\right)^{p}, \quad \ell=1, \ldots, L .
$$

It follows from Lemma 5.3 that the singular supports of the symbols of the operators $\Gamma\left(\mathrm{h}_{1}\right), \Gamma\left(\mathrm{h}_{-1}\right)$ and $\Gamma\left(h_{\ell}\right)$ consist of the points $1,-1$ and of the pairs $\zeta_{\ell}, \bar{\zeta}_{\ell}$, respectively. So we can apply Theorem 3.2 (the localization principle for eigenvalues) to the Hankel operator $\Gamma\left(h_{*}\right)$ with

$$
h_{*}=\mathrm{h}_{1}+\mathrm{h}_{-1}+\sum_{\ell=1}^{L} h_{\ell}
$$

which yields

$$
\Delta_{p}^{ \pm}\left(\Gamma\left(h_{*}\right)\right)=\delta_{p}^{ \pm}\left(\Gamma\left(h_{*}\right)\right)=\Delta_{p}^{ \pm}\left(\Gamma\left(\mathrm{h}_{1}\right)\right)+\Delta_{p}^{ \pm}\left(\Gamma\left(\mathrm{h}_{-1}\right)\right)+\sum_{\ell=1}^{L} \Delta_{p}^{ \pm}\left(\Gamma\left(h_{\ell}\right)\right) .
$$

Now relations (5.9) and (5.10) imply that

$$
\Delta_{p}^{ \pm}\left(\Gamma\left(h_{*}\right)\right)=\delta_{p}^{ \pm}\left(\Gamma\left(h_{*}\right)\right)=\varkappa(\alpha)^{p}\left(\left(\mathrm{~b}_{1}\right)_{ \pm}^{p}+\left(\mathrm{b}_{-1}\right)_{ \pm}^{p}+\sum_{\ell=1}^{L}\left|b_{\ell}\right|^{p}\right) .
$$

Finally, set $g=h-h_{*}$. Using the representation (5.8) and our conditions on $\mathrm{g}_{1}, \mathrm{~g}_{-1}, g_{1}, \ldots, g_{L}$ and applying Theorem 5.6 , we see that $\Gamma(g) \in \mathbf{S}_{p, \infty}^{0}$. Since $\Gamma(h)=$ $\Gamma\left(h_{*}\right)+\Gamma(g)$, it follows from Lemma 2.1 that

$$
\Delta_{p}^{ \pm}(\Gamma(h))=\Delta_{p}^{ \pm}\left(\Gamma\left(h_{*}\right)\right) \quad \text { and } \quad \delta_{p}^{ \pm}(\Gamma(h))=\delta_{p}^{ \pm}\left(\Gamma\left(h_{*}\right)\right) .
$$

Thus by (5.11), we obtain the relations (1.8), (1.9) .

5.3. Spectral asymptotics for Hankel operators in the Hardy space. Here we give an analogue of Theorem 5.7 in terms of the Hankel operators $H(\omega)$ in the space $H^{2}(\mathbb{T})$. They are linked to the operators $\Gamma(h)$ by formulas $(1.3),(1.5)$. Below we consider a class of symbols $\omega$ whose Fourier coefficients satisfy the asymptotic relation (5.8). All necessary calculations have already been done in [14. Here we only state the results. Note that our notation is slightly different from that in [14] because in 14 Hankel operators were considered in a different representation.

We consider a class of functions $\omega(\mu)$ that are smooth on the unit circle except at some finite number of points where they have logarithmic singularities. We 
describe an admissible singularity supposing first that it is located at the point $\mu=1$. Let us introduce an even function $\chi_{0} \in C^{\infty}(\mathbb{R})$ satisfying the condition

$$
\chi_{0}(\theta)= \begin{cases}1 & \text { for }|\theta| \leq c_{1}, \\ 0 & \text { for }|\theta| \geq c_{2},\end{cases}
$$

with $c_{1} \in\left(0, c_{2}\right)$ and sufficiently small $c_{2}$. We accept the following sufficiently general assumption.

Assumption 5.8. Let $\alpha>0$, and let $v_{j, \sigma}(\theta)$ and $u_{j, \sigma}(\theta), j=0,1, \sigma= \pm$, be complex valued $C^{\infty}$ functions of $\theta \in \mathbb{R}$ such that

$$
v_{0,+}(0)=v_{0,-}(0)=: v_{0} .
$$

Then the function $\omega$ is defined by the relation

$$
\omega\left(e^{i \theta}\right)=\sum_{j=0,1} \sum_{\sigma= \pm} v_{j, \sigma}(\theta)\left(-\log |\theta|+u_{j, \sigma}(\theta)\right)^{1-j-\alpha} \mathbb{1}_{\sigma}(\theta) \chi_{0}(\theta), \quad \theta \in(-\pi, \pi] .
$$

Here $c_{2}$ is chosen so small that $\theta=0$ is the only singularity of function (5.13), that is,

$$
-\log |\theta|+u_{j, \sigma}(\theta) \neq 0 \quad \text { if } \quad \theta \in\left[-c_{2}, c_{2}\right]
$$

for $j=0,1, \sigma= \pm$. The branch of the function $z^{j-\alpha}=e^{(j-\alpha) \log z}$ where $z=$ $-\log |\theta|+u_{j, \sigma}(\theta)$ is fixed by the condition

$$
\arg \left(-\log |\theta|+u_{j, \sigma}(\theta)\right) \rightarrow 0 \quad \text { as } \quad \theta \rightarrow 0 .
$$

We emphasize that because of the additional factor $\log |\theta|$, the terms in (5.13) corresponding to $j=0$ are more singular than the terms corresponding to $j=1$. However due to the condition (5.12) the sum of the terms with $j=0$ over $\sigma=+,-$ is essentially an even function of $\theta$. It can be deduced from this fact that the contribution of this sum to the asymptotics of the Fourier coefficients is of the same order as that of the terms corresponding to $j=1$.

For a function $\omega$ satisfying Assumption 5.8, we put

$$
b=(1-\alpha) v_{0}\left(\frac{1}{2}+\frac{1}{2 \pi i}\left(u_{0,+}(0)-u_{0,-}(0)\right)\right)+\frac{1}{2 \pi i}\left(v_{1,+}(0)-v_{1,-}(0)\right) .
$$

If $\omega(\bar{\mu})=\overline{\omega(\mu)}$, then it follows from equality (5.13) that necessarily

$$
v_{0}=\bar{v}_{0}, \quad u_{0,+}(0)=\overline{u_{0,-}(0)}, \quad v_{1,+}(0)=\overline{v_{1,-}(0)} .
$$

In this case $b=\mathrm{b}$ where

$$
\mathrm{b}=(1-\alpha) v_{0}\left(\frac{1}{2}+\frac{1}{\pi} \operatorname{Im} u_{0,+}(0)\right)+\frac{1}{\pi} \operatorname{Im} v_{1,+}(0), \quad v_{0}=\bar{v}_{0},
$$

is real.

From the analytic point of view we rely on the following assertion. 
Theorem 5.9. [14, Theorem 3.2] Under Assumption 5.8, the Fourier coefficients $\widehat{\omega}(j)$ of $\omega(\mu)$ admit the representation

$$
\widehat{\omega}(j)=b j^{-1}(\log j)^{-\alpha}+g(j),
$$

where the coefficient $b$ is given by formula (5.14) and the error term $g(j)$ satisfies the estimates

for all $m \geq 0$.

$$
g^{(m)}(j)=O\left(j^{-1-m}(\log j)^{-\alpha-1}\right), \quad j \rightarrow \infty,
$$

Note that in [14] the asymptotics of $\widehat{\omega}(j)$ was considered for $j \rightarrow-\infty$. In order to translate the results of [14] into the context of this paper, one needs to use the complex conjugation: $\widehat{\omega}_{1}(-j)=\overline{\widehat{\omega}(j)}$ if $\omega_{1}(\mu)=\overline{\omega(\mu)}$.

We emphasize that the leading term of the asymptotics of the Fourier coefficients of the function (5.13) depends on the combination (5.14) only. We also note that without condition (5.12) asymptotics of $\widehat{\omega}(j)$ would be different from (5.16).

Here we state a result about the eigenvalue asymptotics for self-adjoint Hankel operators $H(\omega)$ with symbols having finitely many logarithmic singularities. Thus we suppose that the symbol is a sum of the functions $\omega_{\ell}\left(\mu / \zeta_{\ell}\right)$ where $\zeta_{\ell}$ are distinct points of $\mathbb{T}$ and each $\omega_{\ell}$ satisfies Assumption 5.8. According to the symmetry condition (1.2) if $\operatorname{Im} \zeta_{\ell} \neq 0$, then together with $\omega_{\ell}\left(\mu / \zeta_{\ell}\right)$, the symbol necessarily contains the term $\overline{\omega_{\ell}\left(\bar{\mu} / \zeta_{\ell}\right)}$.

In view of Theorem 5.9, the result below follows directly from Theorem 5.7.

Theorem 5.10. Let functions $\phi_{1}, \phi_{-1}, \omega_{1}, \ldots, \omega_{L}$ satisfy Assumption 5.8. Suppose that

$$
\omega(\mu)=\phi_{1}(\mu)+\phi_{-1}(-\mu)+\sum_{\ell=1}^{L}\left(\omega_{\ell}\left(\mu / \zeta_{\ell}\right)+\overline{\omega_{\ell}\left(\bar{\mu} / \zeta_{\ell}\right)}\right)+\widetilde{\omega}(\mu)
$$

where $\zeta_{1}, \ldots, \zeta_{L} \in \mathbb{T}$ are distinct numbers with $\operatorname{Im} \zeta_{\ell}>0$ and the remainder $\widetilde{\omega} \in L^{2}$ and $P_{+} \widetilde{\omega}$ belongs to the Besov space $B_{1 / \alpha, 1 / \alpha}^{\alpha}(\mathbb{T})$. We assume that the functions $\phi_{1}$, $\phi_{-1}$ and $\widetilde{\omega}$ satisfy the symmetry condition (1.2). Let the numbers $b_{1}, \ldots, b_{L}$ be the asymptotic coefficients for the functions $\omega_{1}, \ldots, \omega_{L}$, defined by (5.14), and let $\mathrm{b}_{1}$, $\mathrm{b}_{-1}$ be the coefficients for $\phi_{1}, \phi_{-1}$, defined by (5.15). Finally, let the coefficient $a^{ \pm}$ be given by (1.9). Then the Hankel operator $H(\omega)$ is compact and its eigenvalues have the asymptotic behavior

$$
\lambda_{n}^{ \pm}(H(\omega))=a^{ \pm} n^{-\alpha}+o\left(n^{-\alpha}\right)
$$

as $n \rightarrow \infty$.

We refer to the book [8], Appendix 2, for the precise definition of Besov classes. Note also that the conditions on the remainder $\widetilde{\omega}$ can be stated (see [14]) in a more explicit although less sharp form. For example, it suffices to suppose that

$$
\widetilde{\omega}(\mu)=\widetilde{\phi}_{1}(\mu)+\widetilde{\phi}_{-1}(-\mu)+\sum_{\ell=1}^{L}\left(\widetilde{\omega}_{\ell}\left(\mu / \zeta_{\ell}\right)+\overline{\widetilde{\omega}_{\ell}\left(\bar{\mu} / \zeta_{\ell}\right)}\right)
$$


where $\widetilde{\phi}_{1}, \widetilde{\phi}_{-1}, \widetilde{\omega}_{1}, \ldots, \widetilde{\omega}_{L}$ satisfy Assumption 5.8 for some $\beta>\alpha$.

Observe that the function $\omega$ in (5.13) is unbounded if $\alpha<1$. Nevertheless according to Theorem 5.10 the corresponding operator $H(\omega)$ is compact. This is of course consistent with the Hartman theorem (see [8], Chapter 1.5) which guarantees that $H(\omega)$ is compact if $\omega \in \operatorname{VMO}(\mathbb{T})$ (the class of functions of vanishing mean oscillation).

\section{Spectral asymptotics for Hankel operators in $L^{2}\left(\mathbb{R}_{+}\right)$}

The main result of this section is stated as Theorem 6.5 where kernels $\mathbf{h}(t)$ are singular both for $t \rightarrow \infty$ and for $t \rightarrow 0$. We also consider (see Theorem 6.8) kernels with singularities at points $t_{0}>0$ (instead of $\left.t_{0}=0\right)$.

6.1. Basic definitions. Integral Hankel operators $\boldsymbol{\Gamma}(\mathbf{h})$ in the space $L^{2}\left(\mathbb{R}_{+}\right)$are formally defined by the relation

$$
(\boldsymbol{\Gamma}(\mathbf{h}) \mathbf{u})(t)=\int_{0}^{\infty} \mathbf{h}(t+s) \mathbf{u}(s) d s, \quad \mathbf{u} \in C_{0}^{\infty}\left(\mathbb{R}_{+}\right),
$$

where $\mathbf{h} \in L_{\text {loc }}^{1}\left(\mathbb{R}_{+}\right)$; this function is called the kernel of the Hankel operator $\boldsymbol{\Gamma}(\mathbf{h})$. Under the assumptions below the operator $\boldsymbol{\Gamma}(\mathbf{h})$ are compact. Of course the operator $\boldsymbol{\Gamma}(\mathbf{h})$ is self-adjoint if and only if the function $\mathbf{h}(t)$ is real valued.

Similarly to the discrete case, bounded Hankel operators $\boldsymbol{\Gamma}(\mathbf{h})$ are unitarily equivalent to the operators $\mathbf{H}(\boldsymbol{\omega})$ defined by formula (3.3) in the Hardy space $H^{2}(\mathbb{R})$ :

$$
\Phi \mathbf{H}(\boldsymbol{\omega}) \Phi^{*}=\boldsymbol{\Gamma}(\mathbf{h}) \quad \text { if } \quad \mathbf{h}(t)=\frac{1}{\sqrt{2 \pi}} \widehat{\boldsymbol{\omega}}(t) \quad \text { for } t>0,
$$

where $\Phi$ is the Fourier transform (3.2). The Fourier transform $\widehat{\omega}$ of $\omega \in L^{\infty}(\mathbb{R})$ should in general be understood in the sense of distributions (for example, on the Schwartz class $\mathcal{S}^{\prime}(\mathbb{R})$ ) and the precise meaning of (6.1) is given by the equation

$$
\left(\mathbf{H}(\boldsymbol{\omega}) \Phi^{*} \mathbf{u}, \Phi^{*} \mathbf{u}\right)=(\boldsymbol{\Gamma}(\mathbf{h}) \mathbf{u}, \mathbf{u}), \quad \mathbf{u} \in C_{0}^{\infty}\left(\mathbb{R}_{+}\right) .
$$

A function $\boldsymbol{\omega}(x)$ satisfying the second equality (6.1) is known as a symbol of the Hankel operator $\boldsymbol{\Gamma}(\mathbf{h})$.

In the discrete case, the spectral asymptotics of $\Gamma(h)$ is determined by the behavior of the sequence $h(j)$ as $j \rightarrow \infty$. In the continuous case, the behavior of the kernel $\mathbf{h}(t)$ for $t \rightarrow \infty$ and for $t \rightarrow 0$ as well as the singularities of $\mathbf{h}(t)$ at points $t_{0}>0$ contribute to the spectral properties of $\boldsymbol{\Gamma}(\mathbf{h})$.

6.2. Previous results. We fix two functions $\chi_{0}, \chi_{\infty} \in C^{\infty}\left(\mathbb{R}_{+}\right)$such that

$$
\chi_{0}(x)=\left\{\begin{array}{ll}
1 & \text { for }|x| \leq c_{1}, \\
0 & \text { for }|x| \geq c_{2},
\end{array} \quad \chi_{\infty}(x)= \begin{cases}0 & \text { for }|x| \leq C_{1}, \\
1 & \text { for }|x| \geq C_{2},\end{cases}\right.
$$

for some $0<c_{1}<c_{2}<1$ and $1<C_{1}<C_{2}$, and define the model kernels

$$
\mathbf{q}_{0}(t)=\chi_{0}(t) t^{-1}(\log (1 / t))^{-\alpha}, \quad \mathbf{q}_{\infty}(t)=\chi_{\infty}(t) t^{-1}(\log t)^{-\alpha}, \quad t>0 .
$$


As usual, the coefficient $\varkappa(\alpha)$ is given by (1.10).

Theorem 6.1. [12, Theorem 3.1] Let $\alpha>0$. Then

$$
\lambda_{n}^{+}\left(\boldsymbol{\Gamma}\left(\mathbf{q}_{0}\right)\right)=\varkappa(\alpha) n^{-\alpha}+o\left(n^{-\alpha}\right) \quad \text { and } \quad \lambda_{n}^{+}\left(\boldsymbol{\Gamma}\left(\mathbf{q}_{\infty}\right)\right)=\varkappa(\alpha) n^{-\alpha}+o\left(n^{-\alpha}\right)
$$

as $n \rightarrow \infty$. Moreover,

$$
\lambda_{n}^{-}\left(\boldsymbol{\Gamma}\left(\mathbf{q}_{0}\right)\right)=o\left(n^{-\alpha}\right) \quad \text { and } \quad \lambda_{n}^{-}\left(\boldsymbol{\Gamma}\left(\mathbf{q}_{\infty}\right)\right)=o\left(n^{-\alpha}\right) .
$$

Let us construct symbols corresponding to the kernels (6.2).

Lemma 6.2. [13, Lemma 6.3] Let $\boldsymbol{\omega}_{0}$ and $\boldsymbol{\omega}_{\infty}$ be defined by

$$
\omega_{0}(x)=2 i \int_{0}^{\infty} \mathbf{q}_{0}(t) \sin (x t) d t, \quad \boldsymbol{\omega}_{\infty}(x)=2 i \int_{0}^{\infty} \mathbf{q}_{\infty}(t) \sin (x t) d t, \quad x \in \mathbb{R},
$$

where $\mathbf{q}_{0}(t)$ and $\mathbf{q}_{\infty}(t)$ are given by (6.2) with $\alpha \geq 0$. Then $\boldsymbol{\omega}_{0}, \boldsymbol{\omega}_{\infty} \in L^{\infty}(\mathbb{R})$ and $\omega_{0} \in C^{\infty}(\mathbb{R}), \omega_{\infty} \in C^{\infty}\left(\mathbb{R}_{*} \backslash\{0\}\right)$. For $t>0$, we have

$$
\mathbf{q}_{0}(t)=\frac{1}{\sqrt{2 \pi}} \widehat{\boldsymbol{\omega}}_{0}(t) \quad \text { and } \quad \mathbf{q}_{\infty}(t)=\frac{1}{\sqrt{2 \pi}} \widehat{\boldsymbol{\omega}}_{\infty}(t) .
$$

The assertion below is a particular case of our general result (Theorem 5.1 in [13]) on the asymptotics of singular values of integral Hankel operators, needed in the present text.

Theorem 6.3. Let $\alpha>0$, let $\rho \in \mathbb{R}, \rho \neq 0$, and let $\mathbf{b} \in \mathbb{C}$ be arbitrary. If

$$
\mathbf{h}(t)=2 \operatorname{Re}\left(\mathbf{b} e^{-i \rho t}\right) \mathbf{q}_{\infty}(t)
$$

then

$$
s_{n}(\boldsymbol{\Gamma}(\mathbf{h}))=2^{\alpha} \varkappa(\alpha)|\mathbf{b}| n^{-\alpha}+o\left(n^{-\alpha}\right) .
$$

Using the symmetry principle, we get the following result.

Theorem 6.4. Let the function $\mathbf{h}(t)$ be the same as in Theorem 6.3, Then

$$
\lambda_{n}^{ \pm}(\boldsymbol{\Gamma}(\mathbf{h}))=\varkappa(\alpha)|\mathbf{b}| n^{-\alpha}+o\left(n^{-\alpha}\right) .
$$

Proof. Let the symbol $\omega_{\infty}$ be defined by (6.3) and let

$$
\boldsymbol{\omega}(x)=\mathbf{b} \boldsymbol{\omega}_{\infty}(x-\rho)+\overline{\mathbf{b}} \boldsymbol{\omega}_{\infty}(x+\rho) .
$$

Then $\widehat{\boldsymbol{\omega}}(t)=\sqrt{2 \pi} \mathbf{h}(t)$ for $t>0$, and hence the operators $\boldsymbol{\Gamma}(\mathbf{h})$ and $\mathbf{H}(\boldsymbol{\omega})$ are unitarily equivalent. By Lemma 6.2, the singular support of the symbol $\boldsymbol{\omega}$ consists of the pair of points $\rho,-\rho$. Therefore by the symmetry principle (Theorem 4.3) we have

$$
\Delta_{p}^{+}(\boldsymbol{\Gamma}(\mathbf{h}))=\Delta_{p}^{-}(\boldsymbol{\Gamma}(\mathbf{h}))=\frac{1}{2} \Delta_{p}(\boldsymbol{\Gamma}(\mathbf{h})),
$$

and similarly for the lower limits. The asymptotic relation (6.4) for the singular values can be equivalently rewritten as $\Delta_{p}(\boldsymbol{\Gamma}(\mathbf{h}))=2 \varkappa(\alpha)^{p}|\mathbf{b}|^{p}$, and thus we obtain

$$
\Delta_{p}^{+}(\boldsymbol{\Gamma}(\mathbf{h}))=\Delta_{p}^{-}(\boldsymbol{\Gamma}(\mathbf{h}))=\varkappa(\alpha)^{p}|\mathbf{b}|^{p}
$$

and similarly for the lower limits. This yields (6.5). 
Note that Theorems 6.3 and 6.4 are the analogues of Theorems 5.4 and 5.5 in the continuous case. The following result concerning the error term is the analogue of Theorem 5.6. Below $\langle x\rangle=\sqrt{1+|x|^{2}}$.

Theorem 6.5. Let $\alpha>0$ and let $M=M(\alpha)$ be the integer given by (5.6). Let $\mathbf{g}$ be a complex valued function in $L_{\mathrm{loc}}^{\infty}\left(\mathbb{R}_{+}\right)$; if $\alpha \geq 1 / 2$, suppose also that $\mathbf{g} \in C^{M}\left(\mathbb{R}_{+}\right)$. Assume that for all $m=0, \ldots, M$, we have

$$
\mathbf{g}^{(m)}(t)=o\left(t^{-1-m}\langle\log t\rangle^{-\alpha}\right)
$$

as $t \rightarrow \infty$ and as $t \rightarrow 0$. Pick any $\rho \in \mathbb{R}$ and put $\mathbf{g}_{\rho}(t)=e^{-i \rho t} \mathbf{g}(t)$. Then $s_{n}\left(\boldsymbol{\Gamma}\left(\mathbf{g}_{\rho}\right)\right)=o\left(n^{-\alpha}\right)$.

6.3. Asymptotics of eigenvalues. Our main result concerns real kernels $\mathbf{h}(t)$ that are singular at $t=0$ and contain several oscillating terms at infinity. The assertion below is the analogue of Theorem 5.7, and its proof follows the same steps.

Theorem 6.6. Let $\alpha>0$, let $\rho_{1}, \ldots, \rho_{L}$ be distinct positive numbers, and let $\mathbf{b}_{0}, \mathbf{b}_{1}, \ldots, \mathbf{b}_{L}, \mathbf{b}_{\infty}$ as well as $\psi_{1}, \ldots, \psi_{L}$ be any real numbers. Let the number $M=$ $M(\alpha)$ be given by (5.6). Suppose that $\mathbf{h} \in L_{\text {loc }}^{\infty}\left(\mathbb{R}_{+}\right)$if $\alpha<1 / 2$ and $\mathbf{h} \in C^{M}\left(\mathbb{R}_{+}\right)$ if $\alpha \geq 1 / 2$. Assume that

$$
\begin{aligned}
& \mathbf{h}(t)=\mathbf{b}_{\infty} t^{-1}(\log t)^{-\alpha}+\mathbf{g}_{\infty}(t)+2 \sum_{\ell=1}^{L}\left(\mathbf{b}_{\ell} t^{-1}(\log t)^{-\alpha}+\mathbf{g}_{\ell}(t)\right) \cos \left(\rho_{\ell} t-\psi_{\ell}\right) \quad t \geq 2, \\
& \mathbf{h}(t)=\mathbf{b}_{0} t^{-1}(\log (1 / t))^{-\alpha}+\mathbf{g}_{0}(t), \quad t \leq 1 / 2,
\end{aligned}
$$

where the error terms $\mathbf{g}_{\infty}, \mathbf{g}_{1}, \ldots, \mathbf{g}_{L}$ obey the estimates (6.6) as $t \rightarrow \infty$ and $\mathbf{g}_{0}$ obeys these estimates as $t \rightarrow 0$. Then the eigenvalues of the integral Hankel operator $\boldsymbol{\Gamma}(\mathbf{h})$ satisfy the asymptotic relation

$$
\lambda_{n}^{ \pm}(\boldsymbol{\Gamma}(\mathbf{h}))=\mathbf{a}^{ \pm} n^{-\alpha}+o\left(n^{-\alpha}\right)
$$

where

$$
\mathbf{a}^{ \pm}=\varkappa(\alpha)\left(\left(\mathbf{b}_{0}\right)_{ \pm}^{1 / \alpha}+\left(\mathbf{b}_{\infty}\right)_{ \pm}^{1 / \alpha}+\sum_{\ell=1}^{L}\left|\mathbf{b}_{\ell}\right|^{1 / \alpha}\right)^{\alpha}
$$

and the coefficient $\varkappa(\alpha)$ is given by (1.10).

Proof. We first consider every term in the right-hand sides of (6.7) and (6.8) separately. Recall that the functions $\mathbf{q}_{0}(t)$ and $\mathbf{q}_{\infty}(t)$ are defined by formulas (6.2). Put

and

$$
\mathbf{h}_{0}(t)=\mathbf{b}_{0} \mathbf{q}_{0}(t), \quad \mathbf{h}_{\infty}(t)=\mathbf{b}_{\infty} \mathbf{q}_{\infty}(t)
$$

$$
\mathbf{h}_{\ell}(t)=2 \mathbf{b}_{\ell} \cos \left(\rho_{\ell} t-\psi_{\ell}\right) \mathbf{q}_{\infty}(t)=2 \operatorname{Re}\left(\mathbf{b}_{\ell} e^{i \psi_{\ell}} e^{-i \rho_{\ell} t}\right) \mathbf{q}_{\infty}(t), \quad \ell=1, \ldots, L .
$$


Similarly to Theorem 5.7, we make the reasoning in terms of the functionals $\Delta_{p}^{ \pm}$, $\delta_{p}^{ \pm}$, where $p=1 / \alpha$. By Theorem 6.1, we have

$$
\begin{gathered}
\Delta_{p}^{ \pm}\left(\boldsymbol{\Gamma}\left(\mathbf{h}_{0}\right)\right)=\delta_{p}^{ \pm}\left(\boldsymbol{\Gamma}\left(\mathbf{h}_{0}\right)\right)=\left(\varkappa(\alpha) \mathbf{b}_{0}\right)_{ \pm}^{p}, \\
\Delta_{p}^{ \pm}\left(\boldsymbol{\Gamma}\left(\mathbf{h}_{\infty}\right)\right)=\delta_{p}^{ \pm}\left(\boldsymbol{\Gamma}\left(\mathbf{h}_{\infty}\right)\right)=\left(\varkappa(\alpha) \mathbf{b}_{\infty}\right)_{ \pm}^{p},
\end{gathered}
$$

and by Theorem 6.4, we have

$$
\Delta_{p}^{ \pm}\left(\boldsymbol{\Gamma}\left(\mathbf{h}_{\ell}\right)\right)=\delta_{p}^{ \pm}\left(\boldsymbol{\Gamma}\left(\mathbf{h}_{\ell}\right)\right)=\left|\varkappa(\alpha) \mathbf{b}_{\ell}\right|^{p}, \quad \ell=1, \ldots, L .
$$

It follows from Lemma 6.2 that the singular supports of the symbols of the operators $\boldsymbol{\Gamma}\left(\mathbf{h}_{0}\right)$ and $\boldsymbol{\Gamma}\left(\mathbf{h}_{\infty}\right)$ consist of the points $\infty$ and 0 , respectively. Lemma 6.2 also implies that the singular supports of the symbols of the operators $\boldsymbol{\Gamma}\left(\mathbf{h}_{\ell}\right)$ consist of the pairs $\left\{-\rho_{\ell}, \rho_{\ell}\right\}$. So we can apply Theorem 3.3 (the localization principle for eigenvalues) to the Hankel operator $\boldsymbol{\Gamma}\left(\mathbf{h}_{*}\right)$ with

$$
\mathbf{h}_{*}=\mathbf{h}_{0}+\mathbf{h}_{\infty}+\sum_{\ell=1}^{L} \mathbf{h}_{\ell}
$$

which yields

$$
\Delta_{p}^{ \pm}\left(\boldsymbol{\Gamma}\left(\mathbf{h}_{*}\right)\right)=\delta_{p}^{ \pm}\left(\boldsymbol{\Gamma}\left(\mathbf{h}_{*}\right)\right)=\Delta_{p}^{ \pm}\left(\boldsymbol{\Gamma}\left(\mathbf{h}_{0}\right)\right)+\Delta_{p}^{ \pm}\left(\boldsymbol{\Gamma}\left(\mathbf{h}_{\infty}\right)\right)+\sum_{\ell=1}^{L} \Delta_{p}^{ \pm}\left(\boldsymbol{\Gamma}\left(\mathbf{h}_{\ell}\right)\right) .
$$

Now relations (6.11) and (6.12) imply that

$$
\Delta_{p}^{ \pm}\left(\boldsymbol{\Gamma}\left(\mathbf{h}_{*}\right)\right)=\delta_{p}^{ \pm}\left(\boldsymbol{\Gamma}\left(\mathbf{h}_{*}\right)\right)=\varkappa(\alpha)^{p}\left(\left(\mathbf{b}_{0}\right)_{ \pm}^{p}+\left(\mathbf{b}_{\infty}\right)_{ \pm}^{p}+\sum_{\ell=1}^{L}\left|\mathbf{b}_{\ell}\right|^{p}\right) .
$$

Finally, put $\mathbf{g}=\mathbf{h}-\mathbf{h}_{*}$. Using representations (6.7), (6.8), our conditions on $\mathbf{g}_{\infty}, \mathbf{g}_{1}, \ldots, \mathbf{g}_{L}, \mathbf{g}_{0}$ and applying Theorem 6.5, we see that $\boldsymbol{\Gamma}(\mathbf{g}) \in \mathbf{S}_{p, \infty}^{0}$. Since $\boldsymbol{\Gamma}(\mathbf{h})=\boldsymbol{\Gamma}\left(\mathbf{h}_{*}\right)+\boldsymbol{\Gamma}(\mathbf{g})$, it follows from Lemma 2.1 that

$$
\Delta_{p}^{ \pm}(\boldsymbol{\Gamma}(\mathbf{h}))=\Delta_{p}^{ \pm}\left(\boldsymbol{\Gamma}\left(\mathbf{h}_{*}\right)\right) \quad \text { and } \quad \delta_{p}^{ \pm}(\boldsymbol{\Gamma}(\mathbf{h}))=\delta_{p}^{ \pm}\left(\boldsymbol{\Gamma}\left(\mathbf{h}_{*}\right)\right) .
$$

Now using (6.14), we obtain the relations (6.9), (6.10).

6.4. Local singularities of the kernel. The localization principle shows that the results on the asymptotics of eigenvalues of different Hankel operators can be combined provided that the singular supports of their symbols are disjoint. This idea has already been illustrated by Theorems 5.4 and 6.6. Here we apply the same arguments to kernels $\mathrm{h}(t)$ satisfying condition (6.7) as $t \rightarrow \infty$ and singular at some positive point.

The effect of local singularities of a kernel on the asymptotics of eigenvalues of the corresponding Hankel operator was studied in [15]. 
Lemma 6.7. [15, Lemma 6.2] Let $t_{0}>0, m \in \mathbb{Z}_{+}$and

$$
\mathbf{f}(t)=\left(t_{0}-t\right)^{m} \quad \text { for } \quad t \leq t_{0}, \quad \mathbf{f}(t)=0 \quad \text { for } \quad t>t_{0} .
$$

Then

$$
\lambda_{n}^{ \pm}(\boldsymbol{\Gamma}(\mathbf{f}))=m ! t_{0}^{m+1}(2 \pi n)^{-m-1}\left(1+O\left(n^{-1}\right)\right), \quad n \rightarrow \infty .
$$

We also note the explicit formula for the symbol $\boldsymbol{\tau}_{m}(x)$ of the operator $\boldsymbol{\Gamma}(\mathbf{f})$ :

$$
\boldsymbol{\tau}_{m}(x)=m !(i x)^{-m-1}\left(e^{i t_{0} x}-\sum_{k=0}^{m} \frac{1}{k !}\left(i t_{0} x\right)^{k}\right), \quad x \in \mathbb{R} .
$$

Obviously, $\boldsymbol{\tau}_{m} \in C^{\infty}(\mathbb{R})$ and $\boldsymbol{\tau}_{m}(x)$ is an oscillating function as $|x| \rightarrow \infty$. Therefore sing supp $\boldsymbol{\tau}_{m}=\{\infty\}$, and hence the symmetry principle (Theorem 4.3) cannot be applied to the operator $\boldsymbol{\Gamma}(\mathbf{f})$. Nevertheless according to (6.16) its spectrum is asymptotically symmetric.

We are now in a position to consider the general case.

Theorem 6.8. Let $t_{0}>0, m \in \mathbb{Z}_{+}$and $\mathrm{b} \in \mathbb{R}$. Set

$$
\mathrm{h}(t)=\mathrm{b} \mathbf{f}(t)+\mathbf{h}(t)
$$

where $\mathbf{f}(t)$ is given by (6.15) and $\mathbf{h}(t)$ satisfies the assumptions of Theorem 6.6 with $\mathbf{b}_{0}=0$ and $\alpha=m+1$. Then the eigenvalues of the integral Hankel operator $\boldsymbol{\Gamma}(\mathrm{h})$ satisfy the asymptotic relation

$$
\lambda_{n}^{ \pm}(\boldsymbol{\Gamma}(\mathrm{h}))=\mathrm{a}^{ \pm} n^{-m-1}+o\left(n^{-m-1}\right)
$$

with

$$
\mathrm{a}^{ \pm}=\left((2 \pi)^{-1} t_{0}(m !|\mathbf{b}|)^{1 / \alpha}+\varkappa(\alpha)^{1 / \alpha}\left(\left(\mathbf{b}_{\infty}\right)_{ \pm}^{1 / \alpha}+\sum_{\ell=1}^{L}\left|\mathbf{b}_{\ell}\right|^{1 / \alpha}\right)\right)^{\alpha}, \quad \alpha=m+1 .
$$

The proof of this result is practically the same as that of Theorem 6.6. The only difference is that the term $\mathbf{h}_{0}(t)$ should be replaced by bf $(t)$ in (6.13).

Observe that we have excluded the term (6.8) singular at $t=0$ in Theorem 6.8 because the corresponding symbol is singular at the same point $x=\infty$ as the function (6.17). In this case one might expect that the contributions of singularities of $\mathrm{h}(t)$ at $t=0$ and $t=t_{0}>0$ are not independent of each other. In any case, our technique does not allow us to treat this situation. Finally, we note that we have chosen $\alpha=m+1$ in Theorem 6.8 since in this case both the local singularity of $\mathrm{h}(t)$ at $t=t_{0}$ and its "tail" as $t \rightarrow \infty$ contribute to the asymptotic coefficient $\mathrm{a}^{ \pm}$ in (6.18).

Similarly to Section 5, Theorems 6.6 and 6.8 can also be reformulated in terms of Hankel operators in the Hardy space $H^{2}(\mathbb{R})$, but we do not dwell upon it here. 


\section{REFERENCES}

[1] M. Sh. Birman, L. S. Koplienko, M. Z. Solomyak, Estimates of the spectrum of a difference of fractional powers of selfadjoint operators. (Russian) Izv. Vyssh. Uchebn. Zaved. Matematika, 3(154) (1975), 3-10.

[2] M. Sh. Birman, M. Z. Solomyak, Spectral theory of selfadjoint operators in Hilbert space. D. Reidel, Dordrecht, 1987.

[3] M. Sh. Birman, M. Z. Solomyak, Compact operators with power asymptotic behavior of the singular numbers. J. Sov. Math. 27 (1984), 2442-2447.

[4] J. S. Howland, Spectral theory of self-adjoint Hankel matrices, Michigan Math. J. 33 (1986), 145-153.

[5] A. V. Megretskit, V. V. Peller, and S. R. Treil, The inverse spectral problem for self-adjoint Hankel operators, Acta Math. 174 (1995), 241-309.

[6] Z. NeHARI, On bounded bilinear forms, Ann. of Math. (2) 65 (1957), 153-162.

[7] N. K. NikOLSKI, Operators, functions, and systems: an easy reading, vol. I: Hardy, Hankel, and Toeplitz, Math. Surveys and Monographs vol. 92, Amer. Math. Soc., Providence, Rhode Island, 2002.

[8] V. PeLler, Hankel operators and their applications, Springer, 2003.

[9] S. R. Power, Hankel operators with discontinuous symbols, Proc. Amer. Math. Soc. 65 (1977), 77-79.

[10] A. Pushnitski, D. YAfaev, Spectral and scattering theory of self-adjoint Hankel operators with piecewise continuous symbols, Journal of Operator Theory 74:2 (2015), 417-455.

[11] A. Pushnitski, D. YAFAev, Sharp estimates for singular values of Hankel operators, Integr. Equ. Oper. Theory, 83: 3 (2015), 393-411.

[12] A. Pushnitski, D. Yafaev, Asymptotic behavior of eigenvalues of Hankel operators, Int. Math. Res. Notices, 2015, no. 22 (2015), 11861-11886.

[13] A. Pushnitski, D. Yafaev, Localization principle for compact Hankel operators, to appear in J. Funct. Analysis, preprint: arXiv:1508.04279.

[14] A. Pushnitski, D. Yafaev, Best rational approximation of functions with logarithmic singularities, preprint: arXiv:1601.00882,

[15] D. R. YAFAEv, Criteria for Hankel operators to be sign-definite, Analysis \& PDE 8 (2015), no. $1,183-221$.

Department of Mathematics, King's College London, Strand, London, WC2R 2LS, U.K.

E-mail address: alexander.pushnitski@kcl.ac.uk

Department of Mathematics, University of Rennes-1, Campus Beaulieu, 35042, Rennes, France

E-mail address: yafaev@univ-rennes1.fr 